J. Nonlinear Var. Anal. 2 (2018), No. 2, pp. 177-202

Available online at http://jnva.biemdas.com

https://doi.org/10.23952/jnva.2.2018.2.06

\title{
A SECOND ORDER FINITE DIFFERENCE SCHEME FOR A VARIABLE INFECTION-STRUCTURED MODEL OF MYCOBACTERIUM MARINUM DYNAMICS IN AQUATIC ANIMALS
}

\author{
AZMY S. ACKLEH ${ }^{1, *}$, KARYN L. SUTTON $^{2}$, TINGTING TANG ${ }^{3}$, LIHONG ZHAO $^{4}$ \\ ${ }^{1}$ Department of Mathematics, University of Louisiana at Lafayette, Lafayette, LA 70504, USA \\ ${ }^{2}$ Institute for Disease Modeling, 3150 139th Avenue Southeast, Bellevue, WA 98005, USA \\ ${ }^{3}$ Department of Applied and Computational Mathematics and Statistics, \\ University of Notre Dame, Notre Dame, IN 46556, USA \\ ${ }^{4}$ Center for Modeling Complex Interactions, University of Idaho, Moscow, ID 83844, USA
}

\begin{abstract}
In this paper, we present a novel second order finite difference scheme for a modified version of a model that was first developed in [4], to study the transmission dynamics of a human TB-like bacterium, Mycobacterium marinum, affecting aquatic animals on a similar scale. Convergence of the finite difference approximation to the unique weak solution of the model is shown. Numerical results confirming the second order convergence of the scheme are presented. The computational advantages of using this high-resolution scheme in comparison to the first order scheme previously used in [4] is illustrated. Keywords. Finite difference method; Second order scheme; Structured population model; Infectious disease.
\end{abstract}

2010 Mathematics Subject Classification. 65L12, 92D30.

\section{INTRODUCTION}

Human tuberculosis (TB), caused by the bacillus Mycobacterium tuberculosis (Mtb), has existed for millennia and remains a major global health problem. In 2015, the world had an estimated 10.4 million new (incident) TB cases, with $60 \%$ of the new cases in India, Indonesia, China, Nigeria, Pakistan and South Africa (listed in descending order of their number of cases) [24]. The vast majority of people infected with Mtb do not present TB symptoms during their lifetime [8, 19, 25], and only a small porportion $(\sim 0.1 \%)$ will develop acute disease $[8,25]$. Although the number of TB deaths fell by $22 \%$ between 2000 and 2015, TB remained one of the top 10 causes of death worldwide in 2015 [24], ranking above HIV/AIDS as one of the leading causes of death from an infectious disease [24].

Mycobacterium marinum $(\mathrm{Mm})$ is one of the closest related species to the Mtb complex, grows much faster, and poses little risk to researchers. Similar to TB, it establishes chronic infections in a variety of fish species over a wide geographic distribution, and survives and multiplies within host macrophages, produces granulomas (the hallmark lesion for $\mathrm{Mtb}$ ), and colonizes hematopoietic tissues present in the spleen, kidneys, and liver [7, 8, 9, 11, 14, 17, 18, 23]. Annually, fish mycobacteriosis costs billions of

\footnotetext{
${ }^{*}$ Corresponding author.

E-mail addresses: ackleh@louisiana.edu (A. S. Ackleh), ksutton@idmod.org (K. L. Sutton), ttang@nd.edu (T. Tang), lihong@uidaho.edu (L. Zhao).

Received December 31, 2017; Accepted April 4, 2018.
}

(C) 2018 Journal of Nonlinear and Variational Analysis 
dollars in combined losses to the wild-caught fisheries, to aquaculture-raised fish, to the aquarium trade, and to research colonies [12, 13, 14].

In the last fifteen years, researchers have developed the Mm-Japanese Medaka (Oryizas latipes) animal infection model $[7,9,11,16,18]$ in order to document and study chronic TB-like infections in fish. As in other chronic infection models, experiments routinely yield substantial variability in organ colonization. The disparity in courses of infection in several of their experiments (unpublished data) was extensive despite all variables (such as dose, virulence of Mm strain, age and health of animals) being near identical. These and other observations provide motivation for the development of a model that accounts for the variability seen in the disease outcomes, in order to build a useful tool in making reasonable predictions or to design control strategies.

A structured model was developed in [4], for the study of Mm transmission dynamics in aquatic animals. In that model, a discrete number of physiological groups were included to reflect differences in individual susceptibility to infection, effectiveness of immune response, feeding behavior, etc. In recent work [5], a noted improvement in the agreement between the model and data was achieved by a simpler but more biologically meaningful approach, which takes phenomenological models of commonly observed courses of infection progression for the intra-host progression rates in fewer cohorts of fish. In this paper, we develop and establish the convergence of a second order finite difference scheme for an alternative formulation of the original model, in which the total population of infected fish is a mixture of subpopulations with different intra-host progression rates. The convergence of an approximation scheme for either the original or the alternative formulation of this model has not been addressed previously. We point out that a second order scheme for a different structured model for the Mm transmission dynamics was developed in [3]. In that model individuals were structured by their size rather than their bacterial load. While there are similarities in the mathematical structure between the model studied here and that in [3], there are some key differences. In particular, the boundary conditions for the two models are very different. This necessitates the development of a different second-order finite difference approximation (that preserves nonnegativity of solutions) and the use of different techniques for establishing a priori estimates for the finite-difference approximations.

In Section 2, we present the bacterial load-structured model of Mm transmission dynamics incorporating intra-host variability that we focus on in this work. The approximation scheme is given in Section 3. A priori estimates for the difference scheme are provided in Section 4. The weak solution for the model is given in Section 5, followed by the establishment of the convergence of the numerical scheme to this weak solution. We then numerically confirm the second-order convergence of the numerical scheme in Section 6.1. Finally, in Section 7, we make our concluding remarks.

\section{VARiable Mm Infection-Structured Model}

We study a modified version of the mathematical model, first developed in [4], of Mm transmission dynamics in an aquatic environment. As in the original, we model the infected fish population as being composed of several subpopulations, each with its own progression function $g\left(x, B_{a}, B_{u}, B_{d}, L ; \beta_{i}\right), i=$ $1, \cdots, M$. In particular, let the density of infected fish having bacterial load $x$ with progression rate $\beta_{i}$ denoted by $I^{i}(t, x)$. In this model, for each $i \in\{1, \ldots, M\}$, we explicitly denote by $p_{i}$ the probability that an infected fish belongs to the subpopulation $I^{i}(x, t)$. Here, $\left\{\beta_{1}, \cdots, \beta_{M}\right\} \in \mathscr{B}^{M} \equiv \Pi_{i=1}^{M} C^{2}\left[x_{\min }, x_{\max }\right]$ and the proportions satisfy $0 \leq p_{i} \leq 1, \sum_{i=1}^{M} p_{i}=1$, and are assumed to be independent of time. We also 
denote the activated $\mathrm{Mm}$ by $B_{a}(t)$, the unactivated $\mathrm{Mm}$ by $B_{u}(t)$, the $\mathrm{Mm}$ residing in the carcasses of dead fish by $B_{d}(t)$, the mosquito larvae carrying $\mathrm{Mm}$ by $L(t)$, and the susceptible fish population by $S(t)$. The (modified) bacterial load-structured model is given by

$$
\begin{aligned}
& \frac{d B_{a}}{d t}=\mathscr{I}(t ; \rho)-\gamma B_{a}-\delta B_{a} Y-\kappa B_{a} \\
& \frac{d B_{u}}{d t}=H\left(t, B_{u}\right)+\gamma B_{a}-c_{L} L^{*} B_{u}-\tilde{\delta} B_{u} Y-\kappa B_{u} \\
& \frac{d B_{d}}{d t}=\mathscr{I}\left(t ; x \mu f_{\mu}\right)-B_{d}\left(c_{1} S+c_{1} \mathscr{I}\left(t ; e^{-\alpha_{1} x}\right)\right)-\zeta B_{d}, \\
& \frac{d L}{d t}=\varepsilon c_{L}\left(L^{*}-L\right) B_{u}-L\left(c_{2} S+c_{2} \mathscr{I}\left(t ; e^{-\alpha_{2} x}\right)\right)-\mu_{L} L \\
& \frac{d S}{d t}=F(Y)-\mu S-v_{1} \delta B_{a} S-v_{2} c_{1} B_{d} S-v_{3} \eta c_{2} L S \\
& \frac{\partial I^{i}(t, x)}{\partial t}+\frac{\partial\left(g\left(x, B_{a}, B_{u}, B_{d}, L ; \beta_{i}\right) I^{i}(t, x)\right)}{\partial x}+\mu f_{\mu}(x) I^{i}(t, x)=0, \quad i=1, \cdots, M,
\end{aligned}
$$

with boundary condition

$$
\left.g\left(x, B_{a}, B_{u}, B_{d}, L ; \beta_{i}\right) I^{i}(t, x)\right|_{x=x_{\min }}=v_{1} \delta B_{a} S+v_{2} c_{1} B_{d} S+v_{3} \eta c_{2} L S, \quad i=1, \cdots, M,
$$

and initial conditions

$$
B_{a}(0)=B_{a}^{0}, \quad B_{u}(0)=B_{u}^{0}, \quad B_{d}(0)=B_{d}^{0}, \quad L(0)=L^{0}, \quad S(0)=S^{0}, \quad I^{i}(0, x)=I^{i, 0} .
$$

Here, the notation $\mathscr{I}(t ; w)$ is defined by

$$
\mathscr{I}(t ; w)=\sum_{i=1}^{M}\left(\int_{x_{\min }}^{x_{\max }} w(x) I^{i}(t, x) d x\right) p_{i},
$$

where $w(x)$ is a continuous (weighting) function. The total infected fish population is then $\mathscr{I}(t ; 1)$ and the total population of fish $Y(t)$ is given by

$$
Y(t)=S(t)+\mathscr{I}(t ; 1)=S(t)+\sum_{i=1}^{M}\left(\int_{x_{\min }}^{x_{\max }} I^{i}(t, x) d x\right) p_{i} .
$$

Thus, the total infected fish population density per bacterial load $x$ at time $t$ is given by

$$
I(t, x)=\sum_{i=1}^{M} I^{i}(t, x) p_{i}
$$

Mm in an activated state $\left(B_{a}(t)\right)$ increases by being shed via defecation by infected fish with rate $\mathscr{I}(t ; \rho)$, and can be lost through deactivation $\gamma B_{a}$, consumption by fish $\delta B_{a} Y$, and consumption by other possible species $\kappa B_{a}$. The number of planktonic $\mathrm{Mm}$ in an unactivated state $B_{u}(t)$ is increased by human activities, such as water run-off from large aquaculture facilities [10, 15, 22] at rate $H\left(t, B_{u}\right)$. Unactivated bacteria may be consumed by mosquito larvae at rate $c_{L} L^{*} B_{u}$, or they may also be consumed by fish at rate $\tilde{\delta} B_{u} Y$, or other species at rate $\kappa B_{u}$. The population of Mm within the carcasses of fish $B_{d}(t)$ increases with rate $\mathscr{I}\left(t ; x \mu f_{\mu}\right)$ as fish die, and is consumed by susceptible and infected fish at rate $B_{d}\left(c_{1} S+\right.$ $c_{1} \mathscr{I}\left(t ; e^{-\alpha_{1} x}\right)$, where the fraction $e^{-\alpha_{1} x}$ is intended to account for an inhibition of feeding rate with increasing bacterial load $x$, a measure of infection severity, as the animal progresses from a chronic to an acute state. The carcasses may also be consumed by other species at rate $\zeta B_{d}$. Mosquito larvae become effective carriers at per capita rate $\varepsilon$ as they consume Mm-laden biofilms $\varepsilon c_{L}\left(L^{*}-L\right) B_{u}$. They 
are lost as they are consumed by fish $L\left(c_{2} S+c_{2} \mathscr{I}\left(t ; e^{-\alpha_{2} x}\right)\right)$ and as they mature into the pupal stage at rate $\mu_{L} L$. The susceptible fish $S(t)$ population increases as fish are born as hatchlings with rate $F(Y)$. This population decreases due to natural death at rate $\mu S$, or due to fish becoming infected by consuming activated planktonic Mm at rate $v_{1} \delta B_{a} S$, Mm-laden carcasses at rate $v_{2} c_{1} B_{d} S$, or carrier mosquito larvae at rate $v_{3} \eta c_{2} L S$. The infection rates constitute the boundary condition of the infected fish, given by (2.2). All of these infection modes may accelerate the progression of infection, or increase the bacterial load within an infected fish, and therefore also appear in the function $g\left(x, B_{a}, B_{u}, B_{d}, L ; \beta_{i}\right)$. The function $f_{\mu}(x)$ scales for the degree to which the death rate is increased in an infected fish according to the bacterial load $x$, or the severity of the fish's infection. Note that all the susceptible fish appear in each of the terms in the boundary condition for infected fish in each group $i$. However, these newly infected fish are increasing only each proportion of the infected fish population at the rate of the infections. For a more detailed explanation and the biological meaning of each of the parameters we refer the reader to [4].

We impose the following regularity conditions on our model parameters throughout the discussion, and let $c$ be a sufficiently large positive constant.

(H1) $0 \leq \rho(x) \leq c$ for $x \in\left[x_{\min }, x_{\max }\right]$.

(H2) The function $H\left(t, B_{u}\right)$ is Lipschitz continuous in $B_{u}$ with Lipschitz constant $c$, and $0<H\left(t, B_{u}\right) \leq$ $c$ for any $\left(t, B_{u}\right) \in[0, T] \times[0, \infty)$.

(H3) The function $f_{\mu}(x)$ is Lipschitz continuous in $x$ with Lipschitz constant $c, 0 \leq \mu f_{\mu}(x) \leq c$ for any $x \in\left[x_{\min }, x_{\max }\right]$, and $0 \leq \mu \leq c$.

(H4) $0 \leq \varepsilon c_{L} L^{*} \leq c$.

(H5) $0 \leq v_{1} \delta, v_{2} c_{1}, v_{3} \eta c_{2} \leq c$.

(H6) The function $F(Y)$ is Lipschitz continuous in $Y$ with Lipschitz constant $c$, and $0 \leq F(Y) \leq c$ for $Y \in[0, \infty)$.

(H7) The function $g\left(x, B_{a}, B_{u}, B_{d}, L ; \beta_{i}\right)$ is Lipschitz continuous in $B_{a}, B_{u}, B_{d}, L$ with Lipschitz constant $c$, and is twice continuously differentiable with respect to $x$. Moreover, we assume that $0<C_{0} \leq$ $g\left(x, B_{a}, B_{u}, B_{d}, L ; \beta_{i}\right) \leq c$ for $i=1, \cdots, M$ and for $\left(x, B_{a}, B_{u}, B_{d}, L\right) \in\left[x_{\min }, x_{\max }\right] \times \Pi_{i=1}^{4}[0, \infty)$.

(H8) $\kappa, \gamma, \delta, \tilde{\delta}, \mu_{L}, c_{1}, c_{2}, \alpha_{1}, \alpha_{2}, \zeta, v_{1}, v_{2}, v_{3}, \eta, \varepsilon, c_{L}$ and $C_{0}$ are nonnegative constants.

(H9) $B_{a}^{0}, B_{u}^{0}, B_{d}^{0}, L^{0}, S^{0}$ are nonnegative constants. The functions $I^{i, 0}$ are nonnegative functions having total variation bounded by $c$ for $i=1, \cdots, M$.

\section{NUMERICAL SCHEME}

We consider a finite mesh of bacterial load sizes $x_{\min }=x_{0}<x_{1}<\cdots<x_{N}=x_{\max }$. We denote by $\Delta x$ the mesh size, so the bacterial load sizes are $x_{j}=x_{0}+j \Delta x$ for $j=0,1, \cdots, N$. We denote by $k$ the number of time steps taken over the finite interval $[0, T]$, so the time points are $t_{k}=k \Delta t$, for $k=0,1, \cdots, K$, and $\Delta t=T / K$. We remark that the scheme is not restricted to a uniform time step or mesh size, although we consider them both to be uniform here for simplicity of presentation.

We denote by $I_{j}^{i, k}$ the numerical approximation of $I^{i}\left(t_{k}, x_{j}\right)$, the density of infected fish per load at time $t_{k}$ of bacterial load $x_{j}$ with intra-host progression rate $\beta_{i}$, where $\left\{\beta_{1}, \cdots, \beta_{M}\right\}=\mathscr{B}^{M}$. We use $S^{k}$ to represent the approximation of the number of susceptible fish, $S\left(t_{k}\right)$, at time $t_{k}$, and $B_{a}^{k}, B_{u}^{k}, B_{d}^{k}, L^{k}$ to denote the approximation of the number of activated bacteria $B_{a}\left(t_{k}\right)$, unactivated bacteria $B_{u}\left(t_{k}\right)$, bacteria from dead infected fish $B_{d}\left(t_{k}\right)$, and carrier mosquito larvae $L\left(t_{k}\right)$ at time $t_{k}$, respectively. Similar notation is used to approximate other model functions and parameters. 
Throughout this paper, the integrals are approximated via the right-hand rectangular rule on the first interval and trapezoid rule for the remaining intervals. We use the following summation operation $\Sigma^{\star}(\cdot)$ to implement the quadrature rule for integrals with respect to $x$

$$
\sum_{j=j_{1}}^{j_{2}}{ }^{\star} u_{j}=\frac{3}{2} u_{j_{1}}+\frac{1}{2} u_{j_{2}}+\sum_{j=j_{1}+1}^{j_{2}-1} u_{j} .
$$

We denote by $\mathscr{I}^{k}(w)$ the approximation of $\mathscr{I}\left(t_{k} ; w\right)$ as

$$
\mathscr{I}^{k}(w)=\sum_{i=1}^{M} \sum_{j=1}^{N} w\left(x_{j}\right) I^{i}\left(t_{k}, x_{j}\right) \Delta x p_{i},
$$

and for illustrative purposes, we have taken $w$ to be a function of $x$ (i.e., $w=w(x)$ ). We denote by $Y^{k}$ the approximation of the total population $Y\left(t_{k}\right)$, which is $Y^{k}=S^{k}+\mathscr{I}^{k}(1)$. We define the finite difference operators

$$
\Delta_{+} I_{j}^{i, k}=I_{j+1}^{i, k}-I_{j}^{i, k}, \quad 0 \leq j \leq N-1, \quad \text { and } \quad \Delta_{-} I_{j}^{i, k}=I_{j}^{i, k}-I_{j-1}^{i, k} \quad 1 \leq j \leq N .
$$

Then, for $k=0, \cdots, K-1, j=1, \cdots, N$, and $i=1, \cdots, M$,

$$
\begin{aligned}
& \frac{B_{a}^{k+1}-B_{a}^{k}}{\Delta t}=\mathscr{I}^{k}(\rho)-\gamma B_{a}^{k+1}-\delta Y^{k} B_{a}^{k+1}-\kappa B_{a}^{k+1}, \\
& \frac{B_{u}^{k+1}-B_{u}^{k}}{\Delta t}=H^{k}+\gamma B_{a}^{k+1}-c_{L} L^{*} B_{u}^{k+1}-\tilde{\delta} B_{u}^{k+1} Y^{k}-\kappa B_{u}^{k+1}, \\
& \frac{B_{d}^{k+1}-B_{d}^{k}}{\Delta t}=\mathscr{I}^{k}\left(x \mu f_{\mu}\right)-B_{d}^{k+1}\left(c_{1} S^{k}+c_{1} \mathscr{I}^{k}\left(e^{-\alpha_{1} x}\right)\right)-\zeta B_{d}^{k+1}, \\
& \frac{L^{k+1}-L^{k}}{\Delta t}=\varepsilon c_{L}\left(L^{*}-L^{k+1}\right) B_{u}^{k+1}-L^{k+1}\left(c_{2} S^{k}+c_{2} \mathscr{I}^{k}\left(e^{-\alpha_{2} x}\right)\right)-\mu_{L} L^{k+1}, \\
& \frac{S^{k+1}-S^{k}}{\Delta t}=F^{k}-\mu S^{k+1}-v_{1} \delta B_{a}^{k} S^{k+1}-v_{2} c_{1} B_{d}^{k} S^{k+1}-v_{3} \eta c_{2} L^{k} S^{k+1}, \\
& \frac{I_{j}^{i, k+1}-I_{j}^{i, k}}{\Delta t}+\frac{J_{j+\frac{1}{2}}^{i, k}-J_{j-\frac{1}{2}}^{i, k}}{\Delta x}+\mu f_{\mu, j} I_{j}^{i, k+1}=0,
\end{aligned}
$$

where $J_{j+\frac{1}{2}}^{i, k}$ is given by

$$
J_{j+\frac{1}{2}}^{i, k}=\left\{\begin{array}{cl}
g_{j}^{i, k} I_{j}^{i, k}+\frac{1}{2}\left(g_{j+1}^{i, k}-g_{j}^{i, k}\right) I_{j}^{i, k} & \\
+\frac{1}{2} g_{j}^{i, k} m m\left(\Delta_{+} I_{j}^{i, k}, \Delta_{-} I_{j}^{i, k}\right) & j=2, \cdots, N-2 \\
g_{j}^{i, k} I_{j}^{i, k} & j=0,1, N-1, N,
\end{array}\right.
$$

and the minmod function $\mathrm{mm}$ is defined by

$$
m m(a, b)=\frac{\operatorname{sign}(a)+\operatorname{sign}(b)}{2} \min (|a|,|b|) .
$$

The boundary condition for the infected fish is approximated by

$$
g_{0}^{i, k+1} I_{0}^{i, k+1}=v_{1} \delta B_{a}^{k+1} S^{k+1}+v_{2} c_{1} B_{d}^{k+1} S^{k+1}+v_{3} \eta c_{2} L^{k+1} S^{k+1}, \quad i=1, \ldots, M .
$$

The initial conditions are given by

$$
B_{a}^{0}=B_{a}(0), \quad B_{u}^{0}=B_{u}(0), \quad B_{d}^{0}=B_{d}(0), \quad L^{0}=L(0), \quad S^{0}=S(0), \quad I_{j}^{i, 0}=I^{i, 0}\left(x_{j}\right) .
$$


The implicit finite difference scheme (3.1) can be rewritten as follows:

$$
\begin{aligned}
B_{a}^{k+1} & =\frac{B_{a}^{k}+\Delta t \mathscr{I}^{k}(\rho)}{1+\Delta t\left(\gamma+\kappa+\delta Y^{k}\right)} \\
B_{u}^{k+1} & =\frac{B_{u}^{k}+\Delta t\left(H^{k}+\gamma B_{a}^{k+1}\right)}{1+\Delta t\left(c_{L} L^{*}+\tilde{\delta} Y^{k}+\kappa\right)} \\
B_{d}^{k+1} & =\frac{B_{d}^{k}+\Delta t \mathscr{I}^{k}\left(x \mu f_{\mu}\right)}{1+\Delta t\left(\zeta+c_{1} S^{k}+c_{1} \mathscr{I}^{k}\left(e^{-\alpha_{1} x}\right)\right)} \\
L^{k+1} & =\frac{L^{k}+\Delta t\left(\varepsilon c_{L} L^{*} B_{u}^{k+1}\right)}{1+\Delta t\left(\varepsilon c_{L} B_{u}^{k+1}+c_{2} S^{k}+c_{2} \mathscr{I}^{k}\left(e^{-\alpha_{2} x}\right)+\mu_{L}\right)} \\
S^{k+1} & =\frac{S^{k}+\Delta t F^{k}}{1+\Delta t\left(\mu+v_{1} \delta B_{a}^{k}+v_{2} c_{1} B_{d}^{k}+v_{3} \eta c_{2} L^{k}\right)} \\
I_{j}^{i, k+1} & =\frac{I_{j}^{i, k}-\frac{\Delta t}{\Delta x}\left(J_{j+\frac{1}{2}}^{i, k}-J_{j-\frac{1}{2}}^{i, k}\right)}{1+\Delta t \mu f_{\mu, j}} .
\end{aligned}
$$

for $i=1, \cdots, M$, with the boundary condition for the infected fish, $I_{0}^{i, k}$ approximated by

$$
I_{0}^{i, k+1}=\frac{1}{g_{0}^{i, k+1}}\left(v_{1} \delta B_{a}^{k+1} S^{k+1}+v_{2} c_{1} B_{d}^{k+1} S^{k+1}+v_{3} \eta c_{2} L^{k+1} S^{k+1}\right)
$$

and initial conditions below

$$
B_{a}^{0}=B_{a}(0), \quad B_{u}^{0}=B_{u}(0), \quad B_{d}^{0}=B_{d}(0), \quad L^{0}=L(0), \quad S^{0}=S(0), \quad I_{j}^{i, 0}=I^{i, 0}\left(x_{j}\right) .
$$

We remark that by (3.4) and (3.6) the quantities on the right-hand side of (3.5) can be computed. We define the $\ell_{1}, \ell_{\infty}$ norms and total variation of $I^{i, k}$ by

$$
\left\|I^{i, k}\right\|_{1}=\sum_{j=1}^{N}\left|I_{j}^{i, k}\right| \Delta x, \quad\left\|I^{i, k}\right\|_{\infty}=\max _{0 \leq j \leq N}\left|I_{j}^{i, k}\right|, \quad T V\left(I^{i, k}\right)=\sum_{j=0}^{N-1}\left|I_{j+1}^{i, k}-I_{j}^{i, k}\right| .
$$

\section{ESTIMATES FOR THE DifFERENCE APPROXIMATIONS}

Let

$$
D_{j}^{i, k}=\left\{\begin{array}{cl}
g_{j}^{i, k}, & j=1, N, \\
\frac{1}{2}\left(g_{j+1}^{i, k}+g_{j}^{i, k}+g_{j}^{i, k} \frac{m m\left(\Delta_{+} I_{j}^{i, k}, \Delta_{-} I_{j}^{i, k}\right)}{\Delta_{-} I_{j}^{i, k}}\right), & j=2, \\
\frac{1}{2}\left(g_{j+1}^{i, k}+g_{j}^{i, k}+g_{j}^{i, k} \frac{m m\left(\Delta_{+} I_{j}^{i, k}, \Delta_{-} I_{j}^{i, k}\right)}{\Delta_{-} I_{j}^{i, k}}\right. & \\
\left.-g_{j-1}^{i, k} \frac{m m\left(\Delta_{-} I_{j}^{i, k}, \Delta_{-} I_{j-1}^{i, k}\right)}{\Delta_{-} I_{j}^{i, k}}\right), & j=3, \ldots, N-2, \\
g_{j}^{i, k}-\frac{1}{2} g_{j-1}^{i, k} \frac{m m\left(\Delta_{-} I_{j}^{i, k}, \Delta_{-} I_{j-1}^{i, k}\right)}{\Delta_{-} I_{j}^{i, k}}, & j=N-1,
\end{array}\right.
$$


and

$$
G_{j}^{i, k}= \begin{cases}\Delta_{-} g_{j}^{i, k}, & j=1, N, \\ \frac{1}{2} \Delta_{+} g_{j}^{i, k}+\Delta_{-} g_{j}^{i, k}, & j=2, \\ \frac{1}{2}\left(\Delta_{+} g_{j}^{i, k}+\Delta_{-} g_{j}^{i, k}\right), & j=3, \ldots, N-2, \\ \frac{1}{2} \Delta_{-} g_{j}^{i, k}, & j=N-1 .\end{cases}
$$

Simple calculations show that

$$
0 \leq D_{j}^{i, k} \leq \frac{3}{2} c, \quad 0 \leq G_{j}^{i, k} \leq \frac{3}{2} c,
$$

and

$$
D_{j}^{i, k}-G_{j}^{i, k}= \begin{cases}g_{j-1}^{i, k}, & j=1, N, \\ \frac{1}{2} g_{j}^{i, k}+\frac{1}{2} g_{j-1}^{i, k}\left(1-\frac{m m\left(\Delta_{-} I_{j}^{i, k}, \Delta_{-} I_{j-1}^{i, k}\right)}{\Delta_{-} I_{j}^{i, k}}\right), & j=N-1, \\ g_{j-1}^{i, k}+\frac{1}{2} g_{j}^{i, k} \frac{m m\left(\Delta_{+} I_{j}^{i, k}, \Delta_{-} I_{j}^{i, k}\right)}{\Delta_{-} I_{j}^{i, k}}, & j=2, \\ \frac{1}{2} g_{j}^{i, k}\left(1+\frac{m m\left(\Delta_{+} I_{j}^{i, k}, \Delta_{-} I_{j}^{i, k}\right)}{\Delta_{-} I_{j}^{i, k}}\right) & \\ \quad+\frac{1}{2} g_{j-1}^{i, k}\left(1-\frac{m m\left(\Delta_{-} I_{j}^{i, k}, \Delta_{-} I_{j-1}^{i, k}\right)}{\Delta_{-} I_{j}^{i, k}}\right), & j=3, \ldots, N-2 .\end{cases}
$$

It is easy to check that

$$
D_{j}^{i, k}-G_{j}^{i, k} \geq 0 .
$$

With the above notation, the last equation of (3.4) can be rewritten as follows for $1 \leq i \leq M, 1 \leq j \leq N$ and $0 \leq k \leq K$ :

$$
\left(1+\Delta t \mu f_{\mu, j}\right) I_{j}^{i, k+1}=\left(1-\frac{\Delta t}{\Delta x} D_{j}^{i, k}\right) I_{j}^{i, k}+\frac{\Delta t}{\Delta x}\left(D_{j}^{i, k}-G_{j}^{i, k}\right) I_{j-1}^{i, k} .
$$

We impose the following CFL-type condition concerning $\Delta t$ and $\Delta x$

(H10) $\frac{3}{2} \frac{\Delta t}{\Delta x} \leq \frac{1}{c}$.

First we show that the difference approximation system has a unique nonnegative solution.

Lemma 4.1. The system (3.1)-(3.3) has a unique nonnegative solution.

Proof. Uniqueness is obvious from Equations (3.4) and (3.5). From (H9), we have that $B_{a}^{0}, B_{u}^{0}, B_{d}^{0}, L^{0}$, $S^{0}$, and $I^{i, 0}$ are nonnegative. Now assume that $B_{a}^{k}, B_{u}^{k}, B_{d}^{k}, L^{k}, S^{k} \geq 0$ and $I_{j}^{i, k} \geq 0$ for $k>0, i=1, \cdots, M$, and $j=0, \cdots, N$. By (H7), (H10), and Equation (4.1), we have

$$
1-\frac{\Delta t}{\Delta x} D_{j}^{i, k} \geq 1-\frac{\Delta t}{\Delta x} \frac{3}{2} c \geq 0 .
$$

Hence, from Equation (3.4), (4.2), (4.3), and assumptions (H1)-(H5), (H8), we have $B_{a}^{k+1}, B_{u}^{k+1}, B_{d}^{k+1}$, $L^{k+1}, S^{k+1} \geq 0$, and $I_{j}^{i, k+1} \geq 0$ for $i=1, \cdots, M, j=0, \cdots, N$. This establishes the result.

In the lemma below we show that $I^{i}$ is bounded in the $\ell_{1}$ norm for $i=1, \ldots, M$, and $B_{a}(t), B_{u}(t), B_{d}(t)$, $L(t), S(t)$ are also bounded. 
Lemma 4.2. Assume that $\Delta t$ satisfies $c \Delta t<1$. Then there exists a positive constant $C_{1}$, independent of $\Delta x$ and $\Delta t$, such that

$$
\left|B_{u}{ }^{k}\right|+\left|B_{a}{ }^{k}\right|+\left|B_{d}{ }^{k}\right|+\left|L^{k}\right|+\left|S^{k}\right|+\sum_{i=1}^{M}\left\|I^{i, k}\right\|_{1} p_{i} \leq C_{1}
$$

for $k=0,1, \ldots, K$.

Proof. First we observe that $S^{k}$ is bounded. In particular, using assumption (H6) we obtain:

$$
\left|S^{k+1}\right|+\Delta t\left(v_{1} \delta B_{a}^{k}+v_{2} c_{1} B_{d}^{k}+v_{3} \eta c_{2} L^{k}\right) S^{k+1} \leq\left|S^{k}\right|+\Delta t F^{k} \leq\left|S^{k}\right|+c \Delta t
$$

Hence, it follows from (4.5) that there exists a constant $C_{5}$ independent of $\Delta x$ and $\Delta t$ such that

$$
\left|S^{k}\right| \leq C_{5}, \quad k=0,1, \ldots, K
$$

Now, from (3.4) and assumptions (H1)-(H4), (H8) we arrive at

$$
\begin{aligned}
\left|B_{a}^{k+1}\right| & \leq\left|B_{a}^{k}\right|+\Delta t \mathscr{I}^{k}(\rho)-\Delta t \gamma\left|B_{a}^{k+1}\right| \\
& \leq|| B_{a}^{k}\left|+\Delta t \sum_{i=1}^{M} \sum_{j=1}^{N^{\star}} \rho\left(x_{j}\right) I_{j}^{i, k} \Delta x p_{i}-\Delta t \gamma\right| B_{a}^{k+1} \mid \\
& \leq\left|B_{a}^{k}\right|+c \Delta t \sum_{i=1}^{M} \sum_{j=1}^{N} I_{j}^{i, k} \Delta x p_{i}-\Delta t \gamma\left|B_{a}^{k+1}\right| \\
& \leq\left|B_{a}^{k}\right|+\frac{3 c}{2} \Delta t \sum_{i=1}^{M}\left\|I^{i, k}\right\|_{1} p_{i}-\Delta t \gamma\left|B_{a}^{k+1}\right|, \\
\left|B_{u}^{k+1}\right| & \leq\left|B_{u}^{k}\right|+\Delta t\left(H^{k}+\gamma\left|B_{a}^{k+1}\right|\right) \leq\left|B_{u}^{k}\right|+c \Delta t+\Delta t \gamma\left|B_{a}^{k+1}\right|, \\
\left|B_{d}^{k+1}\right| & \leq\left|B_{d}^{k}\right|+\Delta t \mathscr{I}^{k}\left(x \mu f_{\mu}\right) \leq\left|B_{d}^{k}\right|+\Delta t \sum_{i=1}^{M} \sum_{j=1}^{N} x_{j} \mu f_{\mu, j} I_{j}^{i, k} \Delta x p_{i} \\
& \leq\left|B_{d}^{k}\right|+\frac{3}{2} c x_{\max } \Delta t \sum_{i=1}^{M}\left\|I^{i, k}\right\|_{1} p_{i}, \\
\left|L^{k+1}\right| & \leq\left|L^{k}\right|+\Delta t \varepsilon c_{L} L^{*}\left|B_{u}^{k+1}\right| \leq\left|L^{k}\right|+c \Delta t\left|B_{u}^{k+1}\right| .
\end{aligned}
$$

We rewrite the last equation in (3.4) as

$$
\left(1+\Delta t \mu f_{\mu, j}\right) I_{j}^{i, k+1}=I_{j}^{i, k}-\frac{\Delta t}{\Delta x}\left(J_{j+\frac{1}{2}}^{i, k}-J_{j-\frac{1}{2}}^{i, k}\right)
$$

Multiplying (4.8) by $\Delta x$ and $p_{i}$ and summing over $j=1, \cdots, N$ and $i=1, \cdots, M$, we get

$$
\sum_{i=1}^{M} \sum_{j=1}^{N}\left(1+\Delta t \mu f_{\mu, j}\right) I_{j}^{i, k+1} \Delta x p_{i}=\sum_{i=1}^{M} \sum_{j=1}^{N} I_{j}^{i, k} \Delta x p_{i}-\Delta t \sum_{i=1}^{M} \sum_{j=1}^{N}\left(J_{j+\frac{1}{2}}^{i, k}-J_{j-\frac{1}{2}}^{i, k}\right) p_{i}
$$


By boundary condition (3.3) and assumption (H3), (H7), and (H8), we obtain

$$
\begin{aligned}
\sum_{i=1}^{M}\left\|I^{i, k+1}\right\|_{1} p_{i} & \leq \sum_{i=1}^{M}\left\|I^{i, k}\right\|_{1} p_{i}-\Delta t \sum_{i=1}^{M} \sum_{j=1}^{N}\left(J_{j+\frac{1}{2}}^{i, k}-J_{j-\frac{1}{2}}^{i, k}\right) p_{i} \\
& =\sum_{i=1}^{M}\left\|I^{i, k}\right\|_{1} p_{i}-\Delta t \sum_{i=1}^{M}\left(g_{N}^{i, k} I_{N}^{i, k}-g_{0}^{i, k} I_{0}^{i, k}\right) p_{i} \\
& \leq \sum_{i=1}^{M}\left\|I^{i, k}\right\|_{1} p_{i}+\Delta t \sum_{i=1}^{M} g_{0}^{i, k} I_{0}^{i, k} p_{i} \\
& =\sum_{i=1}^{M}\left\|I^{i, k}\right\|_{1} p_{i}+\Delta t \sum_{i=1}^{M}\left(v_{1} \delta B_{a}^{k}+v_{2} c_{1} B_{d}^{k}+v_{3} \eta c_{2} L^{k}\right) S^{k} p_{i} \\
& =\sum_{i=1}^{M}\left\|I^{i, k}\right\|_{1} p_{i}+\Delta t\left(v_{1} \delta B_{a}^{k}+v_{2} c_{1} B_{d}^{k}+v_{3} \eta c_{2} L^{k}\right) S^{k} .
\end{aligned}
$$

Adding (4.5), (4.7), and (4.9) yields

$$
\begin{aligned}
& \left|B_{a}^{k+1}\right|+\left|B_{u}^{k+1}\right|+\left|B_{d}^{k+1}\right|+\left|L^{k+1}\right|+\left|S^{k+1}\right|+\sum_{i=1}^{M}\left\|I^{i, k+1}\right\|_{1} p_{i} \\
& \leq\left|B_{a}^{k}\right|+\left|B_{u}^{k}\right|+\left|B_{d}^{k}\right|+\left|L^{k}\right|+\left|S^{k}\right|+\left(1+\frac{3}{2} c \Delta t+\frac{3}{2} x_{\max } c \Delta t\right) \sum_{i=1}^{M}\left\|I^{i, k}\right\|_{1} p_{i} \\
& \quad+c \Delta t\left|B_{u}^{k+1}\right|+2 c \Delta t+\Delta t \sum_{i=1}^{M}\left(v_{1} \delta B_{a}^{k}+v_{2} c_{1} B_{d}^{k}+v_{3} \eta c_{2} L^{k}\right)\left(S^{k}-S^{k+1}\right) \\
& \leq\left|B_{a}^{k}\right|+\left|B_{u}^{k}\right|+\left|B_{d}^{k}\right|+\left|L^{k}\right|+\left|S^{k}\right|+\left(1+\frac{3}{2} c \Delta t+\frac{3}{2} x_{\max } c \Delta t\right) \sum_{i=1}^{M}\left\|I^{i, k}\right\|_{1} p_{i} \\
& \quad+c \Delta t\left|B_{u}^{k+1}\right|+2 c \Delta t+C_{5} \Delta t \sum_{i=1}^{M}\left(v_{1} \delta B_{a}^{k}+v_{2} c_{1} B_{d}^{k}+v_{3} \eta c_{2} L^{k}\right) \\
& \leq\left|B_{a}^{k}\right|+\left|B_{u}^{k}\right|+\left|B_{d}^{k}\right|+\left|L^{k}\right|+\left|S^{k}\right|+\left(1+\frac{3}{2} c \Delta t+\frac{3}{2} x_{\max } c \Delta t\right) \sum_{i=1}^{M}\left\|I^{i, k}\right\|_{1} p_{i} \\
& \quad+c \Delta t\left|B_{u}^{k+1}\right|+2 c \Delta t+c C_{5} \Delta t\left(\left|B_{a}^{k}\right|+\left|B_{d}^{k}\right|+\left|L^{k}\right|\right) .
\end{aligned}
$$

Therefore

$$
\begin{aligned}
& \left|B_{a}^{k+1}\right|+(1-c \Delta t)\left|B_{u}^{k+1}\right|+\left|B_{d}^{k+1}\right|+\left|L^{k+1}\right|+\left|S^{k+1}\right|+\sum_{i=1}^{M}\left\|I^{i, k+1}\right\|_{1} p_{i} \\
& \leq\left(1+c C_{5} \Delta t\right)\left(\left|B_{a}^{k}\right|+\left|B_{d}^{k}\right|+\left|L^{k}\right|\right)+\left|B_{u}^{k}\right|+\left|S^{k}\right|+\left(1+\frac{3}{2} c \Delta t+\frac{3}{2} x_{\max } c \Delta t\right) \sum_{i=1}^{M}\left\|I^{i, k}\right\|_{1} p_{i}+2 c \Delta t,
\end{aligned}
$$

which implies

$$
\begin{aligned}
& (1-c \Delta t)\left(\left|B_{a}^{k+1}\right|+\left|B_{u}^{k+1}\right|+\left|B_{d}^{k+1}\right|+\left|L^{k+1}\right|+\left|S^{k+1}\right|+\sum_{i=1}^{M}\left\|I^{i, k+1}\right\|_{1} p_{i}\right) \\
& \leq(1+\widehat{C} \Delta t)\left(\left|B_{a}^{k}\right|+\left|B_{u}^{k}\right|+\left|B_{d}^{k}\right|+\left|L^{k}\right|+\left|S^{k}\right|+\sum_{i=1}^{M}\left\|I^{i, k}\right\|_{1} p_{i}\right)+2 c \Delta t
\end{aligned}
$$

where $\widehat{C} \geq \max \left\{c C_{5}, 2 c\left(1+x_{\max }\right)\right\}$. The result follows from the last inequality. 
For the remainder of the paper, we let $\mathbb{D}_{1}=\left[x_{\min }, x_{\max }\right] \times\left[0, C_{1}\right] \times\left[0, C_{1}\right] \times\left[0, C_{1}\right] \times\left[0, C_{1}\right], \mathbb{D}_{2}=$ $\left[0, C_{1}\right] \times\left[0, C_{1}\right] \times\left[0, C_{1}\right] \times\left[0, C_{1}\right], \mathbb{D}_{3}=\left[x_{\min }, x_{\max }\right] \times[0, T]$. Also, for notational convenience we set $g^{i}\left(x, B_{a}, B_{u}, B_{d}, L\right)=g\left(x, B_{a}, B_{u}, B_{d}, L ; \beta_{i}\right)$. Following the regularity assumption on $\beta_{i}$ and assumption (H7), we have that $g^{i}$ is twice differentiable with respect to $x$ and Lipschitz continuous with respect to $B_{a}, B_{u}, B_{d}$ and $L$ with Lipschitz constant $c$. Next we establish a bound on the infinity norms of the approximations $I_{j}^{i, k}$.

Lemma 4.3. There exists a positive constant $C_{2}$, independent of $\Delta x$ and $\Delta t$, such that

$$
\left\|I^{i, k}\right\|_{\infty} \leq C_{2}
$$

for $k=0,1, \ldots, K$ and $i=1, \ldots, M$.

Proof. If the maximum is achieved on the left boundary, $\left\|I^{i, k+1}\right\|_{\infty}=\left|I_{0}^{i, k+1}\right|$, then by the boundary condition (3.3), Lemma 4.2, (H5), (H7), and (4.6), we have

$$
\begin{aligned}
\left\|I^{i, k+1}\right\|_{\infty} & =\frac{1}{g_{0}^{i, k+1}}\left(v_{1} \delta B_{a}^{k+1}+v_{2} c_{1} B_{d}^{k+1}+v_{3} \eta c_{2} L^{k+1}\right) S^{k+1} \\
& \leq \frac{c\left(B_{a}^{k+1}+B_{d}^{k+1}+L^{k+1}\right) S^{k+1}}{g_{0}^{i, k+1}} \\
& \leq \frac{c}{C_{0}}\left(B_{a}^{k+1}+B_{d}^{k+1}+L^{k+1}\right) S^{k+1} \\
& \leq \frac{3 c C_{1} C_{5}}{C_{0}}
\end{aligned}
$$

Otherwise, suppose $\left\|I^{i, k+1}\right\|_{\infty}=\left|I_{j_{0}}^{i, k+1}\right|$, where $1 \leq j_{0} \leq N$. According to (4.3) we have

$$
\left(1+\Delta t \mu f_{\mu, j_{0}}\right) I_{j_{0}}^{i, k+1}=\left(1-\frac{\Delta t}{\Delta x} D_{j_{0}}^{i, k}\right) I_{j_{0}}^{i, k}+\frac{\Delta t}{\Delta x}\left(D_{j_{0}}^{i, k}-G_{j_{0}}^{i, k}\right) I_{j_{0}-1}^{i, k}
$$

By (H3), (4.2) and the fact that $I_{j_{0}-1}^{i, k+1} \leq I_{j_{0}}^{i, k+1}$, we reach

$$
I_{j_{0}}^{i, k+1} \leq\left(1-\frac{\Delta t}{\Delta x} D_{j_{0}}^{i, k}\right) I_{j_{0}}^{i, k}+\frac{\Delta t}{\Delta x}\left(D_{j_{0}}^{i, k}-G_{j_{0}}^{i, k}\right) I_{j_{0}-1}^{i, k} \leq I_{j_{0}}^{i, k}-\frac{\Delta t}{\Delta x} G_{j_{0}}^{i, k} I_{j_{0}}^{i, k}
$$

which gives us

$$
\left\|I^{i, k+1}\right\|_{\infty} \leq\left\|I^{i, k}\right\|_{\infty}+\frac{\Delta t}{\Delta x}\left|G_{j_{0}}^{i, k}\right|\left\|I^{i, k}\right\|_{\infty} .
$$

Note that for $1 \leq j \leq N,\left|G_{j}^{i, k+1}\right| \leq \frac{3}{2} \max _{1 \leq j \leq N}\left|g_{j}^{i, k}-g_{j-1}^{i, k}\right|$, hence

$$
\begin{aligned}
\left\|I^{i, k+1}\right\|_{\infty} & \leq\left\|I^{i, k}\right\|_{\infty}+\frac{3}{2} \frac{\Delta t}{\Delta x} \max _{1 \leq j \leq N}\left|g_{j-1}^{i, k}-g_{j}^{i, k}\right|\left\|I^{i, k}\right\|_{\infty} \\
& \leq\left\|I^{i, k}\right\|_{\infty}+\frac{3 \Delta t}{2} \max _{\mathbb{D}_{1}}\left|g_{x}^{i}\left(x, B_{a}, B_{u}, B_{d}, L\right)\right|\left\|I^{i, k}\right\|_{\infty} \\
& =\left(1+\frac{3 \Delta t}{2} \tilde{C}\right)\left\|I^{i, k}\right\|_{\infty},
\end{aligned}
$$

where $\tilde{C}=\max _{\mathbb{D}_{1}}\left|g_{x}^{i}\left(x, B_{a}, B_{u}, B_{d}, L\right)\right|$. Thus,

$$
\left\|I^{i, k+1}\right\|_{\infty} \leq \max \left(\frac{3 c C_{1} C_{5}}{C_{0}}, \frac{2+3 \Delta t \tilde{C}}{2}\left\|I^{i, k}\right\|_{\infty}\right) .
$$

This implies $\left\|I^{i, k+1}\right\|_{\infty} \leq C_{2}$ for an appropriate $C_{2}$. 
From Lemmas 4.2, 4.3 and system (3.1), it follows that there exists a positive constant $C_{3}$ such that

$$
\left|\frac{B_{a}^{k+1}-B_{a}^{k}}{\Delta t}\right| \leq C_{3}, \quad\left|\frac{B_{u}^{k+1}-B_{u}^{k}}{\Delta t}\right| \leq C_{3}, \quad\left|\frac{B_{d}^{k+1}-B_{d}^{k}}{\Delta t}\right| \leq C_{3}, \quad\left|\frac{L^{k+1}-L^{k}}{\Delta t}\right| \leq C_{3}, \quad\left|\frac{S^{k+1}-S^{k}}{\Delta t}\right| \leq C_{3} .
$$

Lemma 4.4. There exists a positive constant $C_{4}$, independent of $\Delta x$ and $\Delta t$, such that $T V\left(I^{i, k}\right) \leq C_{4}$, for $k=0,1, \ldots, K$ and $i=1, \ldots, M$.

Proof. From (4.3), we have

$$
\begin{aligned}
& \left(1+\Delta t \mu f_{\mu, j+1}\right) I_{j+1}^{i, k+1}-\left(1+\Delta t \mu f_{\mu, j}\right) I_{j}^{i, k+1} \\
& =\left(1-\frac{\Delta t}{\Delta x} D_{j+1}^{i, k}\right) I_{j+1}^{i, k}+\frac{\Delta t}{\Delta x}\left(D_{j+1}^{i, k}-G_{j+1}^{i, k}\right) I_{j}^{i, k}-\left(1-\frac{\Delta t}{\Delta x} D_{j}^{i, k}\right) I_{j}^{i, k}-\frac{\Delta t}{\Delta x}\left(D_{j}^{i, k}-G_{j}^{i, k}\right) I_{j-1}^{i, k} .
\end{aligned}
$$

Adding, subtracting and regrouping terms in an appropriate way, we have

$$
\begin{aligned}
& \left(1+\Delta t \mu f_{\mu, j+1}\right)\left(I_{j+1}^{i, k+1}-I_{j}^{i, k+1}\right) \\
= & \left(I_{j+1}^{i, k}-I_{j}^{i, k}\right)-\frac{\Delta t}{\Delta x} D_{j+1}^{i, k}\left(I_{j+1}^{i, k}-I_{j}^{i, k}\right)+\frac{\Delta t}{\Delta x}\left(D_{j}^{i, k}-G_{j}^{i, k}\right)\left(I_{j}^{i, k}-I_{j-1}^{i, k}\right) \\
& -\frac{\Delta t}{\Delta x}\left(G_{j+1}^{i, k}-G_{j}^{i, k}\right) I_{j}^{i, k}-\Delta t \mu\left(f_{\mu, j+1}-f_{\mu, j}\right) I_{j}^{i, k+1} . \\
= & \left(1-\frac{\Delta t}{\Delta x} D_{j+1}^{i, k}\right)\left(I_{j+1}^{i, k}-I_{j}^{i, k}\right)+\frac{\Delta t}{\Delta x}\left(D_{j}^{i, k}-G_{j}^{i, k}\right)\left(I_{j}^{i, k}-I_{j-1}^{i, k}\right) \\
& -\frac{\Delta t}{\Delta x}\left(G_{j+1}^{i, k}-G_{j}^{i, k}\right) I_{j}^{i, k}-\Delta t \mu\left(f_{\mu, j+1}-f_{\mu, j}\right) I_{j}^{i, k+1} .
\end{aligned}
$$

Applying the absolute value, summing over $j=1, \cdots, N-1$, then adding the term $\left(1+\Delta t \mu f_{\mu, 1}\right) \mid I_{1}^{i, k+1}$ $I_{0}^{i, k+1} \mid$ on both sides and using the facts that $1 \leq\left|1+\Delta t \mu f_{\mu, j}\right|, 1-\frac{\Delta t}{\Delta x} D_{j+1}^{i, k}>0$ and $D_{j}^{i, k}-G_{j}^{i, k}>0$, we have

$$
\begin{aligned}
T V\left(I^{i, k+1}\right) \leq & \left(1+\Delta t \mu f_{\mu, 1}\right)\left|I_{1}^{i, k+1}-I_{0}^{i, k+1}\right|+\sum_{j=1}^{N-1}\left|I_{j+1}^{i, k}-I_{j}^{i, k}\right| \\
& +\frac{\Delta t}{\Delta x} \sum_{j=1}^{N-1}\left(-D_{j+1}^{i, k}\left|I_{j+1}^{i, k}-I_{j}^{i, k}\right|+\left(D_{j}^{i, k}-G_{j}^{i, k}\right)\left|I_{j}^{i, k}-I_{j-1}^{i, k}\right|\right) \\
& +\frac{\Delta t}{\Delta x} \sum_{j=1}^{N-1}\left|G_{j+1}^{i, k}-G_{j}^{i, k}\right| I_{j}^{i, k}+\Delta t \sum_{j=1}^{N-1} \mu\left|f_{\mu, j+1}-f_{\mu, j}\right| I_{j}^{i, k+1} \\
= & \left(1+\Delta t \mu f_{\mu, 1}\right)\left|I_{1}^{i, k+1}-I_{0}^{i, k+1}\right|+\sum_{j=1}^{N-1}\left|I_{j+1}^{i, k}-I_{j}^{i, k}\right| \\
& +\frac{\Delta t}{\Delta x}\left(-g_{N}^{i, k}\left|I_{N}^{i, k}-I_{N-1}^{i, k}\right|+g_{1}^{i, k}\left|I_{1}^{i, k}-I_{0}^{i, k}\right|\right)-\frac{\Delta t}{\Delta x} \sum_{j=1}^{N-1} G_{j}^{i, k}\left|I_{j}^{i, k}-I_{j-1}^{i, k}\right| \\
& +\frac{\Delta t}{\Delta x} \sum_{j=1}^{N-1}\left|G_{j+1}^{i, k}-G_{j}^{i, k}\right| I_{j}^{i, k}+\Delta t \sum_{j=1}^{N-1} \mu\left|f_{\mu, j+1}-f_{\mu, j}\right| I_{j}^{i, k+1} \\
\leq & \left(1+\Delta t \mu f_{\mu, 1}\right)\left|I_{1}^{i, k+1}-I_{0}^{i, k+1}\right|+\sum_{j=1}^{N-1}\left|I_{j+1}^{i, k}-I_{j}^{i, k}\right|+\frac{\Delta t}{\Delta x} g_{1}^{i, k}\left|I_{1}^{i, k}-I_{0}^{i, k}\right| \\
& +\frac{\Delta t}{\Delta x} \sum_{j=1}^{N-1}\left|G_{j}^{i, k}\right|\left|I_{j}^{i, k}-I_{j-1}^{i, k}\right|+\frac{\Delta t}{\Delta x} \sum_{j=1}^{N-1}\left|G_{j+1}^{i, k}-G_{j}^{i, k}\right| I_{j}^{i, k} \\
& +\Delta t \sum_{j=1}^{N-1} \mu\left|f_{\mu, j+1}-f_{\mu, j}\right| I_{j}^{i, k+1} .
\end{aligned}
$$


Next, we estimate each term on the right-hand side of the last inequality in (4.11) individually. For the first term, incorporating (4.3), (H3), (H7), the mean value theorem, and Lemma 4.3 we obtain

$$
\begin{aligned}
& \left(1+\Delta t \mu f_{\mu, 1}\right)\left|I_{1}^{i, k+1}-I_{0}^{i, k+1}\right| \\
& =\left|\left(1-\frac{\Delta t}{\Delta x} g_{1}^{i, k}\right) I_{1}^{i, k}+\frac{\Delta t}{\Delta x} g_{0}^{i, k} I_{0}^{i, k}-\left(1+\Delta t \mu f_{\mu, 1}\right) I_{0}^{i, k+1}\right| \\
& \leq\left(1-\frac{\Delta t}{\Delta x} g_{1}^{i, k}\right)\left|I_{1}^{i, k}-I_{0}^{i, k}\right|+\frac{\Delta t}{\Delta x}\left|g_{1}^{i, k}-g_{0}^{i, k}\right| I_{0}^{i, k}+\left|I_{0}^{i, k+1}-I_{0}^{i, k}\right|+\Delta t \mu f_{\mu, 1} I_{0}^{i, k+1} \\
& \leq\left(1-\frac{\Delta t}{\Delta x} g_{1}^{i, k}\right)\left|I_{1}^{i, k}-I_{0}^{i, k}\right|+\Delta t \tilde{C} C_{2}+\left|I_{0}^{i, k+1}-I_{0}^{i, k}\right|+\Delta t c C_{2} .
\end{aligned}
$$

For the fourth term, by the definition of $G_{j}^{i, k}$ and assumption (H7), we have

$$
\left|G_{j}^{i, k}\right| \leq \frac{3}{2} \max _{1 \leq j \leq N_{i}}\left|g_{j}^{i, k}-g_{j-1}^{i, k}\right| \leq \frac{3}{2} \max _{\mathbb{D}_{1}}\left|g_{x}^{i}\right| \Delta x \leq \frac{3}{2} \tilde{C} \Delta x
$$

which gives us

$$
\frac{\Delta t}{\Delta x} \sum_{j=1}^{N-1}\left|G_{j}^{i, k}\right|\left|I_{j}^{i, k}-I_{j-1}^{i, k}\right| \leq \frac{3 \tilde{C}}{2} T V\left(I^{i, k}\right) \Delta t .
$$

For the fifth term, applying (H7), the mean value theorem and Lemma 4.2 - 4.3, we have

$$
\begin{aligned}
\frac{\Delta t}{\Delta x} \sum_{j=1}^{N-1}\left|G_{j+1}^{i, k}-G_{j}^{i, k}\right| I_{j}^{i, k} & =\frac{\Delta t}{\Delta x} \sum_{j=3}^{N-3}\left|G_{j+1}^{i, k}-G_{j}^{i, k}\right| I_{j}^{i, k}+\frac{\Delta t}{\Delta x} \sum_{j=1,2, N-2, N-1}\left|G_{j+1}^{i, k}-G_{j}^{i, k}\right| I_{j}^{i, k} \\
& \leq \frac{\Delta t}{\Delta x} \sum_{j=3}^{N-3} \frac{1}{2}\left|\left(g_{j+2}^{i, k}-2 g_{j+1}^{i, k}+g_{j}^{i, k}\right)+\left(g_{j+1}^{i, k}-2 g_{j}^{i, k}+g_{j-1}^{i, k}\right)\right| I_{j}^{i, k} \\
& +\frac{\Delta t}{\Delta x} 8\left\|I^{i, k}\right\|_{\infty} \max _{0 \leq j \leq N-1}\left|g_{j+1}^{i, k}-g_{j}^{i, k}\right| \\
& \leq \frac{1}{2} \frac{\Delta t}{\Delta x} \sum_{j=3}^{N-3} I_{j}^{i, k}\left(\left|g_{x}^{i}\left(\xi_{j, 1}\right)-g_{x}^{i}\left(\xi_{j, 2}\right)\right| \Delta x+\left|g_{x}^{i}\left(\xi_{j, 2}\right)-g_{x}^{i}\left(\xi_{j, 3}\right)\right| \Delta x\right) \\
& +\frac{\Delta t}{\Delta x} 8 \Delta x\left\|I^{i, k}\right\|_{\infty} \max _{\mathbb{D}_{1}}\left|g_{x}^{i}\left(x, B_{a}, B_{u}, B_{d}, L\right)\right| \\
& \leq \Delta t \sum_{j=3}^{N-3} I_{j}^{i, k}\left(\max _{\mathbb{D}_{1}}\left|g_{x x}^{i}\left(x, B_{a}, B_{u}, B_{d}, L\right)\right| \Delta x\right)+8 \Delta t \tilde{C}\left\|I^{i, k}\right\|_{\infty} \\
& \leq \Delta t \bar{C}\left\|I^{i, k}\right\|_{1}+8 \Delta t \tilde{C}\left\|I^{i, k}\right\|_{\infty} \\
& \leq \Delta t \bar{C} C_{1}+8 \Delta t \tilde{C} C_{2},
\end{aligned}
$$

where $\bar{C}=\max _{\mathbb{D}_{1}}\left|g_{x x}^{i}\left(x, B_{a}, B_{u}, B_{d}, L\right)\right|$. For the last term, we use (H3), (H8), the mean value theorem, and Lemma 4.3 to realize that

$$
\Delta t \sum_{j=1}^{N-1} \mu\left|f_{\mu, j+1}-f_{\mu, j}\right| I_{j}^{i, k+1} \leq \Delta t \sum_{j=1}^{N-1} \mu(c \Delta x) I_{j}^{i, k+1} \leq \Delta t \mu c\left\|I^{i, k+1}\right\|_{1} \leq \Delta t \mu c C_{1} .
$$

Substituting these estimates back into (4.11), we have for $i=1, \cdots, M$,

$$
T V\left(I^{i, k+1}\right) \leq\left(1+\frac{3}{2} \tilde{C} \Delta t\right) T V\left(I^{i, k}\right)+\left|I_{0}^{i, k+1}-I_{0}^{i, k}\right|+\Delta t\left(\bar{C} C_{1}+9 \tilde{C} C_{2}+c C_{2}+\mu c C_{1}\right) .
$$


Next we will consider the term $\left|I_{0}^{i, k+1}-I_{0}^{i, k}\right|$.

$$
\begin{aligned}
& \left|I_{0}^{i, k+1}-I_{0}^{i, k}\right| \\
= & \left|\frac{v_{1} \delta B_{a}^{k+1} S^{k+1}+v_{2} c_{1} B_{d}^{k+1} S^{k+1}+v_{3} \eta c_{2} L^{k+1} S^{k+1}}{g_{0}^{i, k+1}}-\frac{v_{1} \delta B_{a}^{k} S^{k}+v_{2} c_{1} B_{d}^{k} S^{k}+v_{3} \eta c_{2} L^{k} S^{k}}{g_{0}^{i, k}}\right| \\
\leq & \left|\frac{v_{1} \delta B_{a}^{k+1} S^{k+1} g_{0}^{i, k}-v_{1} \delta B_{a}^{k} S^{k} g_{0}^{i, k+1}}{g_{0}^{i, k} g_{0}^{i, k+1}}\right|+\left|\frac{v_{2} c_{1} B_{d}^{k+1} S^{k+1} g_{0}^{i, k}-v_{2} c_{1} B_{d}^{k} S^{k} g_{0}^{i, k+1}}{g_{0}^{i, k} g_{0}^{i, k+1}}\right| \\
& +\left|\frac{v_{3} \eta c_{2}^{i} L^{k+1} S^{k+1} g_{0}^{i, k}-v_{3} \eta c_{2} L^{k} S^{k} g_{0}^{i, k+1}}{g_{0}^{i, k} g_{0}^{i, k+1}}\right| \\
= & A_{1}+A_{2}+A_{3},
\end{aligned}
$$

where $A_{1}, A_{2}$ and $A_{3}$ are the first, second and third terms on the right-hand side of the above inequality, respectively. Adding and subtracting some terms yields

$$
A_{1} \leq \frac{v_{1} \delta B_{a}^{k} S^{k+1}}{g_{0}^{i, k} g_{0}^{i, k+1}}\left|g_{0}^{i, k+1}-g_{0}^{i, k}\right|+\frac{v_{1} \delta B_{a}^{k+1}}{g_{0}^{i, k}}\left|S^{k+1}-S^{k}\right|+\frac{v_{1} \delta S^{k}}{g_{0}^{i, k}}\left|B_{a}^{k+1}-B_{a}^{k}\right| .
$$

By assumption (H7) and Lemma 4.2, we obtain

$$
\begin{aligned}
\left|g_{0}^{i, k+1}-g_{0}^{i, k}\right| & \leq c\left(\left|B_{a}^{k+1}-B_{a}^{k}\right|+\left|B_{u}^{k+1}-B_{u}^{k}\right|+\left|B_{d}^{k+1}-B_{d}^{k}\right|+\left|L^{k+1}-L^{k}\right|\right) \\
& \leq 4 c C_{3} \Delta t .
\end{aligned}
$$

Thus,

$$
A_{1} \leq \frac{c C_{1} C_{5}}{C_{0}{ }^{2}} 4 c C_{3} \Delta t+\frac{c C_{1}}{C_{0}} C_{3} \Delta t+\frac{c C_{5}}{C_{0}} C_{3} \Delta t \leq C_{7} \Delta t
$$

Similarly, enlarging $C_{7}$ if needed, we get the same bound for term $A_{2}$ and $A_{3}$. Therefore,

$$
\left|I_{0}^{i, k+1}-I_{0}^{i, k}\right| \leq 3 C_{7} \Delta t .
$$

Substituting the above estimate back into (4.12), we have for $i=1, \cdots, M$,

$$
T V\left(I^{i, k+1}\right) \leq\left(1+\frac{3 \tilde{C}}{2} \Delta t\right) T V\left(I^{i, k}\right)+M \Delta t,
$$

where $M=3 C_{7}+\bar{C} C_{1}+9 \tilde{C} C_{2}+c C_{2}+\mu c C_{1}$. This implies $T V\left(I^{i, k+1}\right) \leq C_{4}$ for large enough $C_{4}$.

The next lemma shows the numerical approximations satisfy a Lipschitz-type condition in $t$.

Lemma 4.5. There exists a positive constant $C_{8}$, independent of $\Delta x$ and $\Delta t$, such that for any integers $k_{1}>k_{2} \geq 0$

$$
\sum_{j=1}^{N}\left|\frac{I_{j}^{i, k_{1}}-I_{j}^{i, k_{2}}}{\Delta t}\right| \Delta x \leq C_{8}\left(k_{1}-k_{2}\right)
$$

for $i=1, \ldots, M$.

Proof. Multiplying (4.8) by $\Delta x$, summing over $j=1, \cdots, N$, and rearranging terms, we obtain

$$
\sum_{j=1}^{N} \frac{I_{j}^{i, k+1}-I_{j}^{i, k}}{\Delta t} \Delta x=-\sum_{j=1}^{N} \mu f_{\mu, j} I_{j}^{i, k+1} \Delta x-\sum_{j=1}^{N}\left(J_{j+\frac{1}{2}}^{i, k}-J_{j-\frac{1}{2}}^{i, k}\right)
$$


Applying the absolute value, we obtain

$$
\begin{aligned}
\sum_{j=1}^{N} \frac{\left|I_{j}^{i, k+1}-I_{j}^{i, k}\right|}{\Delta t} \Delta x & \leq \sum_{j=1}^{N} \mu f_{\mu, j} I_{j}^{i, k+1} \Delta x+\sum_{j=1}^{N}\left|J_{j+\frac{1}{2}}^{i, k}-J_{j-\frac{1}{2}}^{i, k}\right| \\
& =\sum_{j=1}^{N} \mu f_{\mu, j} I_{j}^{i, k+1} \Delta x+\left|g_{N}^{i, k} I_{N}^{i, k}-g_{0}^{i, k} I_{0}^{i, k}\right| \\
& \leq \sum_{j=1}^{N} \mu f_{\mu, j} I_{j}^{i, k+1} \Delta x+g_{N}^{i, k} I_{N}^{i, k}+g_{0}^{i, k} I_{0}^{i, k} \\
& \leq c\left\|I^{i, k+1}\right\|_{1}+c\left\|I^{i, k}\right\|_{\infty}+\left(n v_{1} \delta B_{a}^{k}+v_{2} c_{1} B_{d}^{k}+v_{3} \eta c_{2} L^{k}\right) S^{k} \\
& \leq c\left\|I^{i, k+1}\right\|_{1}+c C_{2}+c C_{5}\left(\left|B_{a}^{k}\right|+\left|B_{d}^{k}\right|+\left|L^{k}\right|\right) \\
& \leq C_{8} .
\end{aligned}
$$

Hence,

$$
\sum_{j=1}^{N} \frac{\left|I_{j}^{i, k_{1}}-I_{j}^{i, k_{2}}\right|}{\Delta t} \Delta x \leq \sum_{k=k_{2}}^{k_{1}} \sum_{j=1}^{N} \frac{\left|I_{j}^{i, k+1}-I_{j}^{i, k}\right|}{\Delta t} \Delta x \leq C_{8}\left(k_{1}-k_{2}\right)
$$

\section{Convergence of the Difference Approximations to a Weak Solution}

We first give the definition of a weak solution to problem (2.1)-(2.2) as follows:

Definition 5.1. A set of functions $B_{a}(t), B_{u}(t), B_{d}(t), L(t), S(t), I^{i}(t, x)(i=1, \cdots, M)$, where $B_{a}, B_{u}$, $B_{d}, L, S \in C[0, T], I^{i} \in B V\left([0, T] \times\left[x_{\min }, x_{\max }\right]\right)$, is called a weak solution to the problem (2.1)-(2.2) if it satisfies:

$$
\begin{aligned}
& B_{a}(t)=B_{a}(0)+\int_{0}^{t}\left[\mathscr{I}(\tau ; \rho)-\gamma B_{a}(\tau)-\delta B_{a}(\tau) Y(\tau)-\kappa B_{a}(\tau)\right] d \tau \\
& B_{u}(t)=B_{u}(0)+\int_{0}^{t}\left[H\left(\tau, B_{u}(\tau)\right)+\gamma B_{a}(\tau)-c_{L} L^{*} B_{u}(\tau)-\tilde{\delta} B_{u}(\tau) Y(\tau)-\kappa B_{u}(\tau)\right] d \tau \\
& B_{d}(t)=B_{d}(0)+\int_{0}^{t}\left[\mathscr{I}\left(\tau ; x \mu f_{\mu}(x)\right)-B_{d}(\tau)\left(c_{1} S(\tau)+c_{1} \mathscr{I}\left(\tau ; e^{-\alpha_{1} x}\right)\right)-\zeta B_{d}(\tau)\right] d \tau \\
& L(t)=L(0)+\int_{0}^{t}\left[\varepsilon c_{L}\left(L^{*}-L(\tau)\right) B_{u}(\tau)-L(\tau)\left(c_{2} S(\tau)+c_{2} \mathscr{I}\left(\tau ; e^{-\alpha_{2} x}\right)\right)-\mu_{L} L(\tau)\right] d \tau \\
& S(t)=S(0)+\int_{0}^{t}\left[F(Y(\tau))-\mu S(\tau)-v_{1} \delta B_{a}(\tau) S(\tau)-v_{2} c_{1} B_{d}(\tau) S(\tau)-v_{3} \eta c_{2} L(\tau) S(\tau)\right] d \tau
\end{aligned}
$$

and

$$
\begin{aligned}
& \int_{x_{\min }}^{x_{\max }} I^{i}(t, x) \phi(t, x) d x \\
= & \int_{x_{\min }}^{x_{\max }} I^{i}(0, x) \phi(0, x) d x-\int_{0}^{t} g\left(x_{\max }, B_{a}(\tau), B_{d}(\tau), L(\tau) ; \beta_{i}\right) I^{i}\left(\tau, x_{\max }\right) \phi\left(\tau, x_{\max }\right) d \tau \\
& +\int_{0}^{t} S(\tau)\left[v_{1} \delta B_{a}(\tau)+v_{2} c_{1} B_{d}(\tau)+v_{3} \eta c_{2} L(\tau)\right] \phi\left(\tau, x_{\min }\right) d \tau \\
& +\int_{0}^{t} \int_{x_{\min }}^{x_{\max }} I^{i}(\tau, x)\left[\phi_{\tau}(\tau, x)+g\left(x, B_{a}(\tau), B_{d}(\tau), L(\tau) ; \beta_{i}\right) \phi_{x}(\tau, x)-\mu f_{\mu}(x) \phi(\tau, x)\right] d x d \tau
\end{aligned}
$$

for each $i=1, \cdots, M, t \in[0, T], x \in\left[x_{\min }, x_{\max }\right]$, and any test function $\phi \in C^{1}\left([0, T] \times\left[x_{\min }, x_{\max }\right]\right)$. 
Following similar notation as in [21], we define a family of functions $\left\{\left(\mathbb{B}_{a}\right)_{\Delta t}\right\},\left\{\left(\mathbb{B}_{u}\right)_{\Delta t}\right\},\left\{\left(\mathbb{B}_{d}\right)_{\Delta t}\right\}$, $\left\{\mathbb{L}_{\Delta t}\right\},\left\{\mathbb{S}_{\Delta t}\right\}$, and $\left\{\mathbb{I}_{\Delta t, \Delta x}^{i}\right\}$, for $i=1, \cdots, M$ by

$$
\begin{array}{ll}
\left(\mathbb{B}_{a}\right)_{\Delta t}(t)=B_{a}^{k-1}+\frac{B_{a}^{k}-B_{a}^{k-1}}{\Delta t}\left(t-t_{k-1}\right), & \left(\mathbb{B}_{u}\right)_{\Delta t}(t)=B_{u}^{k-1}+\frac{B_{u}^{k}-B_{u}^{k-1}}{\Delta t}\left(t-t_{k-1}\right), \\
\left(\mathbb{B}_{d}\right)_{\Delta t}(t)=B_{d}^{k-1}+\frac{B_{d}^{k}-B_{d}^{k-1}}{\Delta t}\left(t-t_{k-1}\right), & \mathbb{L}_{\Delta t}(t)=L^{k-1}+\frac{L^{k}-L^{k-1}}{\Delta t}\left(t-t_{k-1}\right), \\
\mathbb{S}_{\Delta t}(t)=S^{k-1}+\frac{S^{k}-S^{k-1}}{\Delta t}\left(t-t_{k-1}\right), & \mathbb{I}_{\Delta t, \Delta x}^{i}(t, x)=I_{j}^{i, k},
\end{array}
$$

for $x \in\left[x_{j-1}, x_{j}\right), t \in\left[t_{k-1}, t_{k}\right), j=1, \cdots, N, k=1, \cdots, K$. Then by Lemmas 4.2-4.5, the set of functions $\left\{\left(\mathbb{B}_{a}\right)_{\Delta t}\right\},\left\{\left(\mathbb{B}_{u}\right)_{\Delta t}\right\},\left\{\left(\mathbb{B}_{d}\right)_{\Delta t}\right\},\left\{\mathbb{L}_{\Delta t}\right\}$, and $\left\{\mathbb{S}_{\Delta t}\right\}$ are compact in the topology of $C[0, T]$, and $\left\{\mathbb{I}_{\Delta t, \Delta x}^{i}\right\}$ is compact in the topology of $L^{1}\left((0, T) \times\left(x_{\min }, x_{\max }\right)\right)$.

Theorem 5.1. There exist subsequences $\left\{\left(\mathbb{B}_{a}\right)_{\Delta t_{\ell}}\right\} \subset\left\{\left(\mathbb{B}_{a}\right)_{\Delta t}\right\},\left\{\left(\mathbb{B}_{u}\right)_{\Delta t_{\ell}}\right\} \subset\left\{\left(\mathbb{B}_{u}\right)_{\Delta t}\right\},\left\{\left(\mathbb{B}_{d}\right)_{\Delta t_{\ell}}\right\} \subset\left\{\left(\mathbb{B}_{d}\right)_{\Delta t}\right\}$, $\left\{L_{\Delta t_{\ell}}\right\} \subset\left\{L_{\Delta t}\right\}, \quad\left\{S_{\Delta t_{\ell}}\right\} \subset\left\{S_{\Delta t}\right\}$ and $\left\{\mathbb{I}_{\Delta t_{\ell}, \Delta x_{\ell}}^{i}\right\} \subset\left\{\mathbb{I}_{\Delta t, \Delta \mu}^{i}\right\}$ that converge to functions $B_{a}, B_{u}, B_{d}, L, S \in$ $C[0, T]$ and $I^{i} \in B V\left([0, T] \times\left[x_{\min }, x_{\max }\right]\right)$, respectively, for $i=1, \cdots, M$, in the sense that for all $T>0$,

$$
\begin{aligned}
& \max _{t \in[0, T]}\left|\left(\mathbb{B}_{a}\right)_{\Delta t_{\ell}}(t)-B_{a}(t)\right| \rightarrow 0, \\
& \max _{t \in[0, T]}\left|\left(\mathbb{B}_{u}\right)_{\Delta t_{\ell}}(t)-B_{u}(t)\right| \rightarrow 0, \\
& \max _{t \in[0, T]}\left|\left(\mathbb{B}_{d}\right)_{\Delta t_{\ell}}(t)-B_{d}(t)\right| \rightarrow 0, \\
& \max _{t \in[0, T]}\left|\mathbb{L}_{\Delta t_{\ell}}(t)-L(t)\right| \rightarrow 0, \\
& \max _{t \in[0, T]}\left|\mathbb{S}_{\Delta t_{\ell}}(t)-S(t)\right| \rightarrow 0, \\
& \int_{x_{\min }}^{x_{\max }}\left|\mathbb{I}_{\Delta t_{\ell}, \Delta x_{\ell}}^{i}(t, x)-I^{i}(t, x)\right| d x \rightarrow 0, \\
& \int_{0}^{T} \int_{x_{\min }}^{x_{\max }}\left|\mathbb{I}_{\Delta t_{\ell}, \Delta x_{\ell}}^{i}(t, x)-I^{i}(t, x)\right| d x d t \rightarrow 0,
\end{aligned}
$$

as $\ell \rightarrow \infty\left(\right.$ i.e. $\left.\Delta t_{l}, \Delta x_{l} \rightarrow 0\right)$. Furthermore, there exists a constant $C_{9}$ such that the limit functions satisfy

$$
\left\|B_{a}\right\|_{C[0, T]}+\left\|B_{u}\right\|_{C[0, T]}+\left\|B_{d}\right\|_{C[0, T]}+\|L\|_{C[0, T]}+\|S\|_{C[0, T]}+\left\|I^{i}\right\|_{B V\left([0, T] \times\left[x_{\min }, x_{\max }\right]\right)} \leq C_{9} .
$$

Proof. The results for $B_{a}(t), B_{u}(t), B_{d}(t), L(t)$, and $S(t)$ follow from Theorem I.28 (Ascoli's Theorem) (p. 30) in [20]. Furthermore, the results for $I^{i}(t, x)$ follows using similar arguments as in the proof of Lemma 16.7 (p. 276) in [21].

We show in the next theorem that the set of limit functions $B_{a}(t), B_{u}(t), B_{d}(t), L(t), S(t)$, and $I^{i}(t, x)$, $i=1, \cdots, M$, constructed by the difference scheme is a weak solution to problem (2.1)-(2.2).

Theorem 5.2. The set of limit functions $B_{a}(t), B_{u}(t), B_{d}(t), L(t), S(t), I^{i}(t, x)$ defined in Theorem 5.1 is a weak solution of (2.1)-(2.2) and satisfies

$$
\left|B_{a}(t)\right|+\left|B_{u}(t)\right|+\left|B_{d}(t)\right|+|L(t)|+|S(t)|+\sum_{i=1}^{M}\left\|I^{i}(t, \cdot)\right\|_{1} p_{i} \leq C_{1},
$$

and

$$
\left\|I^{i}\right\|_{L^{\infty}\left((0, T) \times\left(x_{\min }, x_{\max }\right)\right)} \leq C_{2}
$$


Proof. Let $\phi \in C^{1}\left([0, T] \times\left[x_{\min }, x_{\max }\right]\right)$ and denote the finite difference approximation of $\phi\left(t_{k}, x_{j}\right)$ by $\phi_{j}^{k}$. Multiplying the last equation of (3.1) by $\phi_{j}^{k+1}$ and rearranging terms, we obtain

$$
\begin{aligned}
I_{j}^{i, k+1} \phi_{j}^{k+1}-I_{j}^{i, k} \phi_{j}^{k}= & I_{j}^{i, k}\left(\phi_{j}^{k+1}-\phi_{j}^{k}\right)-\Delta t \mu f_{\mu, j} I_{j}^{i, k+1} \phi_{j}^{k+1} \\
& +\frac{\Delta t}{\Delta x}\left[J_{j-\frac{1}{2}}^{i, k} \phi_{j-1}^{k+1}-J_{j+\frac{1}{2}}^{i, k} \phi_{j}^{k+1}+J_{j-\frac{1}{2}}^{i, k}\left(\phi_{j}^{k+1}-\phi_{j-1}^{k+1}\right)\right] .
\end{aligned}
$$

Multiplying the above equation by $\Delta x$, summing over $j=1, \cdots, N$, and $k=0, \cdots, K-1$, we have

$$
\begin{aligned}
\sum_{j=1}^{N}\left(I_{j}^{i, K} \phi_{j}^{K}-I_{j}^{i, 0} \phi_{j}^{0}\right) \Delta x= & \sum_{k=0}^{K-1} \sum_{j=1}^{N}\left[I_{j}^{i, k}\left(\phi_{j}^{k+1}-\phi_{j}^{k}\right)-\Delta t \mu f_{\mu, j} I_{j}^{i, k+1} \phi_{j}^{k+1}\right] \Delta x \\
& +\Delta t \sum_{k=0}^{K-1} \sum_{j=1}^{N}\left(J_{j-\frac{1}{2}}^{i, k} \phi_{j-1}^{k+1}-J_{j+\frac{1}{2}}^{i, k} \phi_{j}^{k+1}\right)+\Delta t \sum_{k=0}^{K-1} \sum_{j=1}^{N} J_{j-\frac{1}{2}}^{i, k}\left(\phi_{j}^{k+1}-\phi_{j-1}^{k+1}\right) \\
= & \sum_{k=0}^{K-1} \sum_{j=1}^{N}\left[I_{j}^{i, k}\left(\phi_{j}^{k+1}-\phi_{j}^{k}\right)-\Delta t \mu f_{\mu, j} I_{j}^{i, k+1} \phi_{j}^{k+1}\right] \Delta x \\
& +\Delta t \sum_{k=0}^{K-1}\left(J_{1-\frac{1}{2}}^{i, k} \phi_{0}^{k+1}-J_{N+\frac{1}{2}}^{i, k} \phi_{N}^{k+1}\right)+\Delta t \sum_{k=0}^{K-1} \sum_{j=1}^{N} J_{j-\frac{1}{2}}^{i, k}\left(\phi_{j}^{k+1}-\phi_{j-1}^{k+1}\right) . \\
= & \sum_{k=0}^{K-1} \sum_{j=1}^{N}\left[I_{j}^{i, k}\left(\phi_{j}^{k+1}-\phi_{j}^{k}\right)-\Delta t \mu f_{\mu, j} I_{j}^{i, k+1} \phi_{j}^{k+1}\right] \Delta x \\
& +\Delta t \sum_{k=0}^{K-1}\left(g_{0}^{i, k} I_{0}^{i, k} \phi_{0}^{k+1}-g_{N}^{i, k} I_{N}^{i, k} \phi_{N}^{k+1}\right)+\Delta t \sum_{k=0}^{K-1} \sum_{j=1}^{N} J_{j-\frac{1}{2}}^{i, k}\left(\phi_{j}^{k+1}-\phi_{j-1}^{k+1}\right) .
\end{aligned}
$$

Using equation (3.2), we have

$$
\begin{aligned}
\Delta t \sum_{k=0}^{K-1} \sum_{j=1}^{N} J_{j-\frac{1}{2}}^{i, k}\left(\phi_{j}^{k+1}-\phi_{j-1}^{k+1}\right)= & \Delta t \sum_{k=0}^{K-1}\left[g_{0}^{i, k} I_{0}^{i, k}+g_{1}^{i, k} I_{1}^{i, k}+g_{N-1}^{i, k} I_{N-1}^{i, k}+\sum_{j \in \Omega_{1}} \frac{1}{2}\left(g_{j+1}^{i, k}+g_{j}^{i, k}\right) I_{j}^{i, k}\right. \\
& +\sum_{j \in \Omega_{2}} \frac{1}{2}\left(g_{j+1}^{i, k} I_{j}^{i, k}+g_{j}^{i, k} I_{j+1}^{i, k}\right) \\
& \left.+\sum_{j \in \Omega_{3}}\left(\frac{1}{2} g_{j+1}^{i, k} I_{j}^{i, k}+g_{j}^{i, k} I_{j}^{i, k}-\frac{1}{2} g_{j}^{i, k} I_{j-1}^{i, k}\right)\right]\left(\phi_{j}^{k+1}-\phi_{j-1}^{k+1}\right),
\end{aligned}
$$

where

$$
\begin{gathered}
\Omega_{1}=\left\{2 \leq j \leq N-2: \operatorname{sign}\left(\Delta_{+} I_{j}^{i, k}\right) \operatorname{sign}\left(\Delta_{-} I_{j}^{i, k}\right)=-1 \text { or } 0\right\}, \\
\Omega_{2}=\left\{2 \leq j \leq N-2: \Delta_{-} I_{j}^{i, k} \geq \Delta_{+} I_{j}^{i, k}>0 \text { or } \Delta_{-} I_{j}^{i, k} \leq \Delta_{+} I_{j}^{i, k}<0\right\},
\end{gathered}
$$

and

$$
\Omega_{3}=\left\{2 \leq j \leq N-2: \Delta_{+} I_{j}^{i, k}>\Delta_{-} I_{j}^{i, k}>0 \text { or } \Delta_{+} I_{j}^{i, k}<\Delta_{-} I_{j}^{i, k}<0\right\}
$$


Therefore, we can rewrite (5.5) as

$$
\begin{aligned}
& \sum_{j=1}^{N}\left(I_{j}^{i, K} \phi_{j}^{K}-I_{j}^{i, 0} \phi_{j}^{0}\right) \Delta x=\sum_{k=0}^{K-1} \sum_{j=1}^{N}\left[I_{j}^{i, k}\left(\phi_{j}^{k+1}-\phi_{j}^{k}\right)-\Delta t \mu f_{\mu, j} I_{j}^{i, k+1} \phi_{j}^{k+1}\right] \Delta x \\
& +\Delta t \sum_{k=0}^{K-1}\left(g_{0}^{i, k} I_{0}^{i, k} \phi_{0}^{k+1}-g_{N}^{i, k} I_{N}^{i, k} \phi_{N}^{k+1}\right) \\
& +\Delta t \sum_{k=0}^{K-1}\left[g_{0}^{i, k} I_{0}^{i, k}+g_{1}^{i, k} I_{1}^{i, k}+g_{N-1}^{i, k} I_{N-1}^{i, k}+\sum_{j \in \Omega_{1}} \frac{1}{2}\left(g_{j+1}^{i, k}+g_{j}^{i, k}\right) I_{j}^{i, k}\right. \\
& +\sum_{j \in \Omega_{2}} \frac{1}{2}\left(g_{j+1}^{i, k} I_{j}^{i, k}+g_{j}^{i, k} I_{j+1}^{i, k}\right) \\
& \left.+\sum_{j \in \Omega_{3}}\left(\frac{1}{2} g_{j+1}^{i, k} I_{j}^{i, k}+g_{j}^{i, k} I_{j}^{i, k}-\frac{1}{2} g_{j}^{i, k} I_{j-1}^{i, k}\right)\right]\left(\phi_{j}^{k+1}-\phi_{j-1}^{k+1}\right) \\
& =\sum_{k=0}^{K-1} \sum_{j=1}^{N}\left[I_{j}^{i, k}\left(\phi_{j}^{k+1}-\phi_{j}^{k}\right)-\Delta t \mu f_{\mu, j} I_{j}^{i, k+1} \phi_{j}^{k+1}\right] \Delta x \\
& +\Delta t \sum_{k=0}^{K-1}\left(\left(v_{1} \delta B_{a}^{k}+v_{2} c_{1} B_{d}^{k}+v_{3} \eta c_{2} L^{k}\right) S^{k} \phi_{0}^{k+1}-g_{N}^{i, k} I_{N}^{i, k} \phi_{N}^{k+1}\right) \\
& +\Delta t \sum_{k=0}^{K-1}\left[g_{0}^{i, k} I_{0}^{i, k}+g_{1}^{i, k} I_{1}^{i, k}+g_{N-1}^{i, k} I_{N-1}^{i, k}+\sum_{j \in \Omega_{1}} \frac{1}{2}\left(g_{j+1}^{i, k}+g_{j}^{i, k}\right) I_{j}^{i, k}\right. \\
& +\sum_{j \in \Omega_{2}} \frac{1}{2}\left(g_{j+1}^{i, k} I_{j}^{i, k}+g_{j}^{i, k} I_{j+1}^{i, k}\right) \\
& \left.+\sum_{j \in \Omega_{3}}\left(\frac{1}{2} g_{j+1}^{i, k} I_{j}^{i, k}+g_{j}^{i, k} I_{j}^{i, k}-\frac{1}{2} g_{j}^{i, k} I_{j-1}^{i, k}\right)\right]\left(\phi_{j}^{k+1}-\phi_{j-1}^{k+1}\right) .
\end{aligned}
$$

Multiplying the first five equations of (3.1) by $\Delta t$ and repeating this procedure for $k, k-1, \cdots, 0$, we have

$$
\begin{gathered}
B_{a}^{k+1}=B_{a}^{0}+\Delta t \sum_{\alpha=0}^{k}\left(\mathscr{I}^{\alpha}(\rho)-\gamma B_{a}^{\alpha+1}-\delta Y^{\alpha} B_{a}^{\alpha+1}-\kappa B_{a}^{\alpha+1}\right) \\
=B_{a}^{0}+\Delta t \sum_{\alpha=0}^{k}\left[\sum_{i=1}^{M} \sum_{j=1}^{N^{\star}} \rho\left(x_{j}\right) I_{j}^{i, \alpha} \Delta x p_{i}-\gamma B_{a}^{\alpha+1}\right. \\
\left.-\delta\left(S^{\alpha}+\sum_{i=1}^{M} \sum_{j=1}^{N} I_{j}^{i, \alpha} \Delta x p_{i}\right) B_{a}^{\alpha+1}-\kappa B_{a}^{\alpha+1}\right], \\
B_{u}^{k+1}=B_{u}^{0}+\Delta t \sum_{\alpha=0}^{k}\left(H^{\alpha}+\gamma B_{a}^{\alpha+1}-c_{L} L^{*} B_{u}^{\alpha+1}-\tilde{\delta} B_{u}^{\alpha+1} Y^{\alpha}-\kappa B_{u}^{\alpha+1}\right) \\
=B_{u}^{0}+\Delta t \sum_{\alpha=0}^{k}\left[H\left(t_{\alpha}, B_{u}^{\alpha}\right)+\gamma B_{a}^{\alpha+1}-c_{L} L^{*} B_{u}^{\alpha+1}\right. \\
\left.-\tilde{\delta} B_{u}^{\alpha+1}\left(S^{\alpha}+\sum_{i=1}^{M} \sum_{j=1}^{N} I_{j}^{i, \alpha} \Delta x p_{i}\right)-\kappa B_{u}^{\alpha+1}\right],
\end{gathered}
$$




$$
\begin{aligned}
B_{d}^{k+1}=B_{d}^{0}+\Delta t \sum_{\alpha=0}^{k}\left(\mathscr{I}^{\alpha}\left(x \mu f_{\mu}\right)-B_{d}^{\alpha+1}\left(c_{1} S^{\alpha}+c_{1} \mathscr{I}^{\alpha}\left(e^{-\alpha_{1} x}\right)\right)-\zeta B_{d}^{\alpha+1}\right) \\
=B_{d}^{0}+\Delta t \sum_{\alpha=0}^{k}\left[\sum_{i=1}^{M} \sum_{j=1}^{N^{\star}} x_{j} \mu f_{\mu}\left(x_{j}\right) I_{j}^{i, \alpha} \Delta x p_{i}\right. \\
\left.\quad-B_{d}^{\alpha+1}\left(c_{1} S^{\alpha}+c_{1} \sum_{i=1}^{M} \sum_{j=1}^{N^{\star}} e^{-\alpha_{1} x_{j}} I_{j}^{i, \alpha} \Delta x p_{i}\right)-\zeta B_{d}^{\alpha+1}\right], \\
L^{k+1}=L^{0}+\Delta t \sum_{\alpha=0}^{k}\left(\varepsilon c_{L}\left(L^{*}-L^{\alpha+1}\right) B_{u}^{\alpha+1}-L^{\alpha+1}\left(c_{2} S^{\alpha}+c_{2} \mathscr{I}^{\alpha}\left(e^{-\alpha_{2} x}\right)\right)-\mu_{L} L^{\alpha+1}\right) \\
=L^{0}+\Delta t \sum_{\alpha=0}^{k}\left[\varepsilon c_{L}\left(L^{*}-L^{\alpha+1}\right) B_{u}^{\alpha+1}\right. \\
\left.\quad-L^{\alpha+1}\left(c_{2} S^{\alpha}+c_{2} \sum_{i=1}^{M} \sum_{j=1}^{N} e^{-\alpha_{2} x_{j}} I_{j}^{i, \alpha} \Delta x p_{i}\right)-\mu_{L} L^{\alpha+1}\right], \\
=S^{0}+\Delta t \sum_{\alpha=0}^{k}\left(F^{\alpha}-\mu S^{\alpha+1}-v_{1} \delta B_{a}^{\alpha} S^{\alpha+1}-v_{2} c_{1} B_{d}^{\alpha} S^{\alpha+1}-v_{3} \eta c_{2} L^{\alpha} S^{\alpha+1}\right) \\
=S^{0}+\Delta t \sum_{\alpha=0}^{k}\left[F\left(S^{\alpha}+\sum_{i=1}^{M} \sum_{j=1}^{N} I_{j}^{i, \alpha} \Delta x p_{i}\right)-\mu S^{\alpha+1}-v_{1} \delta B_{a}^{\alpha} S^{\alpha+1}\right. \\
\left.\quad-v_{2} c_{1} B_{d}^{\alpha} S^{\alpha+1}-v_{3} \eta c_{2} L^{\alpha} S^{\alpha+1}\right] .
\end{aligned}
$$

Since $\mathbb{I}_{\Delta t, \Delta x}^{i}$ is piecewise constant, $\left(\mathbb{B}_{a}\right)_{\Delta t},\left(\mathbb{B}_{u}\right)_{\Delta t},\left(\mathbb{B}_{d}\right)_{\Delta t}, \mathbb{L}_{\Delta t}, \mathbb{S}_{\Delta t}$, and $\phi$ is smooth, and the integrals are limits of step functions, taking $N, k \rightarrow \infty$ on (5.6)-(5.8) and using similar arguments to those in Lemma 16.9 (p. 279) in [21], we establish the result.

Remark 5.1. The uniqueness of the weak solution can be shown using arguments in the spirit of those developed in [2] to establish uniqueness of the weak solution for an Mm model where $I^{i}(t, x)$ is structured by size (not by bacterial load). Thus, since the weak solutions are unique then we obtain that the sequence defined in Theorem 1 converges to the unique weak solution of the model.

Note that the scheme given in (3.1)-(3.3) is of second order accuracy in the bacterial load variable $x$ but only first order in the time variable $t$. To obtain an approximation that is second order in time, we apply Richardson extrapolation in the time variable. In particular, we denote by $I_{j}^{i, k}(\Delta t)$ the approximated model solution for infected fish using a given $\Delta t$, and similar notations for all other variables. Let the vector

$$
W^{k}(\Delta t)=\left\{B_{a}^{k}(\Delta t), B_{u}^{k}(\Delta t), B_{d}^{k}(\Delta t), L^{k}(\Delta t), S^{k}(\Delta t), I_{j}^{i, k}(\Delta t)\right\}
$$

denote the solution of the system (3.1)-(3.3) at time $t_{k}$ when the time mesh is $\Delta t$. The following Richardson's extrapolation iteration, $\tilde{W}^{k}$, at time $t_{k}$ provides second order accuracy in time and is given by

$$
\tilde{W}^{k}=2 W^{2 k}\left(\frac{\Delta t}{2}\right)-W^{k}(\Delta t) .
$$

\section{Computational Studies}

In this section, we demonstrate the performance of the second order scheme (SOS) and illustrate its advantages when compared to a first order scheme (FOS) that was previously published in [4]. While the model (2.1) is highly nonlinear and it is not possible to obtain a closed form solution, in Section 
6.1 we construct an exact solution to a simple version of the model with some forcing terms. We then use this exact solution to test the order of convergence of the SOS and FOS methods. In Section 6.2, we consider the approximated solutions of the full model, without any simplifications. We compare solutions computed from the SOS with solutions from the FOS, and clearly demonstrate the improvement in accuracy and time efficiency of the second order method developed here.

6.1. Convergence for a simplified model. First, we consider the following simple version of our model with two forcing terms $f_{1}$ and $f_{2}$ :

$$
\begin{aligned}
& \frac{d B_{a}}{d t}=-\gamma B_{a}-\delta B_{a} Y-\kappa B_{a} \\
& \frac{d B_{u}}{d t}=\gamma B_{a}-c_{L} L^{*} B_{u}-\tilde{\delta} B_{u} Y-\kappa B_{u}, \\
& \frac{d B_{d}}{d t}=-\zeta B_{d}, \\
& \frac{d L}{d t}=-\mu_{L} L \\
& \frac{d S}{d t}=-\mu S-v_{1} \delta B_{a} S+f_{1}, \\
& \frac{\partial I}{\partial t}=-\left(\frac{\partial}{\partial x}\{g I\}+\mu f_{\mu} I\right)+f_{2} .
\end{aligned}
$$

We let

$$
\begin{gathered}
g(x)=\beta x, \\
f_{1}(t)=e^{-t}\left(\mu+v_{1} \delta B_{a}(t)-1\right), \\
f_{2}(t, x)=e^{x-t}\left(\beta+\beta x+\mu f_{\mu}-1\right),
\end{gathered}
$$

and

$$
f_{\mu}(x)=\exp \left(a \frac{x-x_{\min }}{x_{\max }-x_{\min }}\right) .
$$

We set the initial conditions to be $B_{a}(0)=5, B_{u}(0)=3, B_{d}(0)=100, L(0)=10, S(0)=1, I(0, x)=e^{x}$, and the boundary condition to be $g\left(x_{\min }\right) I\left(t, x_{\min }\right)=\delta v_{1}(t) B a(t) S(t)$, with

$$
v_{1}(t)=\frac{\beta x_{\min } \exp \left(x_{\min }\right) \exp \left\{(\kappa+\gamma) t-\delta\left(e^{-t}-1\right)\left(1+e^{x_{\max }}-e^{x_{\min }}\right)\right\}}{B_{a}(0) \delta} .
$$

Note that while $v_{1}$ is a function of $t$ here, the convergence results in this paper still hold. One can verify that the following set of functions

$$
\begin{aligned}
S(t)= & e^{-t}, \\
I(t, x)= & e^{x-t}, \\
B_{a}(t)= & B_{a}(0) \exp \left\{\delta\left(e^{-t}-1\right)\left(1+e^{x_{\max }}-e^{x_{\min }}\right)-(\gamma+\kappa) t\right\}, \\
B_{u}(t)= & B_{u}(0) \exp \left\{\tilde{\boldsymbol{\delta}}\left(e^{-t}-1\right)\left(e^{x_{\max }}-e^{x_{\min }}+1\right)-t\left(\kappa+c_{L} L^{*}\right)\right\} \\
& \quad+B_{a}(0) \gamma \exp \left\{\left(\tilde{\delta} e^{-t}-\delta\right)\left(e^{x_{\max }}-e^{x_{\min }}+1\right)-t\left(\kappa+c_{L} L^{*}\right)\right\} \\
& \quad \int_{0}^{t} \exp \left\{(\delta-\tilde{\delta}) e^{-\tau}\left(e^{x_{\max }}-e^{x_{\min }}+1\right)+\left(c_{L} L^{*}-\gamma\right) \tau\right\} d \tau, \\
B_{d}(t)= & B_{d}(0) e^{-\zeta t}, \\
L(t)= & L(0) e^{-\mu_{L} t},
\end{aligned}
$$

solves the system (6.1) with the above parameter forms. 
The above exact solution is compared to the numerically approximated solution of system (6.1) via the scheme

$$
\begin{aligned}
& B_{a}^{k+1}=\frac{B_{a}^{k}}{1+\Delta t\left(\gamma+\kappa+\delta Y^{k}\right)}, \quad B_{u}^{k+1}=\frac{B_{u}^{k}+\Delta t \gamma B_{a}^{k+1}}{1+\Delta t\left(c_{L} L^{*}+\tilde{\delta} Y^{k}+\kappa\right)}, \\
& B_{d}^{k+1}=\frac{B_{d}^{k}}{1+\Delta t \zeta}, \quad L^{k+1}=\frac{L^{k}}{1+\Delta t \mu_{L}}, \quad S^{k+1}=\frac{S^{k}+\Delta t f_{1}^{k+1}}{1+\Delta t\left(\mu+v_{1} \delta B_{a}^{k}\right)},
\end{aligned}
$$

where $f_{1}^{k+1}=f_{1}\left(t_{k+1}\right)=e^{-t_{k+1}}\left(\mu+v_{1} \delta B_{a}^{k+1}-1\right)$. The boundary condition is given by

$$
g_{0}^{k+1} I_{0}^{k+1}=v_{1} \delta B a^{k+1} S^{k+1} .
$$

For $j=1, \ldots, N$, the approximated density of infected fish at time step $k+1$ is

$$
I_{j}^{k+1}=\frac{I_{j}^{k}-\frac{\Delta t}{\Delta x}\left(J_{j+\frac{1}{2}}^{k}-J_{j-\frac{1}{2}}^{k}\right)+\Delta t f_{2, j}^{k+1}}{1+\Delta t \mu f_{\mu, j}},
$$

where $f_{2, j}^{k+1}=f_{2}\left(t_{k+1}, x_{j}\right)=e^{x_{j}-t_{k+1}}\left(\beta+\beta x_{j}+\mu f_{\mu}\left(x_{j}\right)-1\right)$. A Richardson extrapolation as described in equation (5.9) is applied to scheme (6.2)- (6.4) in the time variable to obtain a second order method in both $x$ and $t$. Note that the CFL condition (H10) is no longer adequate for the simplified model (6.1), since $f_{1}^{k+1}$ and $f_{2, j}^{k+1}$ are (possibly) negative for some $k, j(k \geq 0, j \geq 1)$. Instead, we need

$$
\left\{\begin{array}{l}
S^{k}+\Delta t f_{1}^{k+1} \geq 0 \\
I_{j}^{k}-\frac{\Delta t}{\Delta x}\left(J_{j+\frac{1}{2}}^{k}-J_{j-\frac{1}{2}}^{k}\right)+\Delta t f_{2, j}^{k+1} \geq 0
\end{array}\right.
$$

to ensure the nonnegativity of the approximated solutions. All of the approximated solutions in the following numerical studies are indeed nonnegative in this section.

TABLE 1. Parameter values for the simplified model (6.1).

\begin{tabular}{c|c||c|c||c|c||c|c}
\hline \hline Parameter & Value & Parameter & Value & Parameter & Value & Parameter & Value \\
\hline$\delta$ & $1 / 500$ & $\mu_{L}$ & $1 / 60$ & $L^{*}$ & 20 & $\beta$ & 0.02 \\
$\tilde{\delta}$ & $1 / 500$ & $\kappa$ & $10^{-4}$ & $c_{1}$ & 5 & $\zeta$ & 0.1 \\
$\mu$ & $1 / 1600$ & $\gamma$ & 0.6 & $a$ & 6 & $x_{\min }$ & 1 \\
$x_{\max }$ & 2 & & & & & & \\
\hline \hline
\end{tabular}

We use the parameter values given in Table 1 to compute several approximated solutions of the model (6.1), halving the step size in both $\Delta t$ and $\Delta x$ with each successive iteration. We then calculate the $L_{1}$ error of $I\left(t_{\max }, \cdot\right)$ and the maximum error for $B_{a}(t), B_{u}(t), B_{d}(t), L(t)$, and $S(t)$.

The numerical results using both the FOS and SOS are shown in Table 2 and 3. As expected, we have first order convergence for the FOS and second order convergence for the SOS. In addition, to compare the computational efficiency for both numerical schemes, we provide Table 4 which includes CPU time for both the FOS and SOS schemes implemented on a PC with Intel Core i7-6500U CUP 2.50 Ghz.

From Table 4, the advantages of using the SOS over the FOS are clear. In particular, to obtain solutions having error on the scale of $10^{-4}$, it takes 205 seconds with the FOS, while it takes less than one second using the SOS. This is a time savings of over $99.9 \%$. This is an important point of consideration since calibration of this model to data is key to the relevance and predictive power of its solutions, and hence 
TABLE 2. The error and order of accuracy resulting from the SOS at time $T=1$.

\begin{tabular}{lllllllll}
\hline$t$ & $x$ & \multicolumn{2}{c}{$I(T,:)$} & \multicolumn{2}{c}{$B_{a}(T)$} & \multicolumn{2}{c}{$B_{u}(T)$} \\
$\times 10^{-2}$ & $\times 10^{-2}$ & $L_{1}$ Error & Order & Error & Order & Error & Order \\
\hline 2.0000 & 2.0000 & $1.34 \mathrm{E}-04$ & & $1.94 \mathrm{E}-05$ & & $1.17 \mathrm{E}-07$ \\
1.0000 & 1.0000 & $3.13 \mathrm{E}-05$ & 2.0991 & $4.91 \mathrm{E}-06$ & 1.9852 & $2.96 \mathrm{E}-08$ & 1.9852 \\
0.5000 & 0.5000 & $7.59 \mathrm{E}-06$ & 2.0463 & $1.23 \mathrm{E}-06$ & 1.9921 & $7.45 \mathrm{E}-09$ & 1.9921 \\
0.2500 & 0.2500 & $1.87 \mathrm{E}-06$ & 2.0237 & $3.09 \mathrm{E}-07$ & 1.9959 & $1.87 \mathrm{E}-09$ & 1.9959 \\
\hline \hline$\Delta t$ & $\Delta x$ & \multicolumn{3}{c}{$B_{d}(T)$} & \multicolumn{3}{c}{$L(T)$} & \multicolumn{2}{c}{$S(T)$} \\
$\times 10^{-2}$ & $\times 10^{-2}$ & Error & Order & Error & Order & Error & Order \\
\hline 2.0000 & 2.0000 & $5.79 \mathrm{E}-06$ & \multicolumn{3}{c}{$3.02 \mathrm{E}-09$} & & $1.23 \mathrm{E}-05$ & \\
1.0000 & 1.0000 & $1.45 \mathrm{E}-06$ & 1.9984 & $7.54 \mathrm{E}-10$ & 2.0006 & $3.07 \mathrm{E}-06$ & 1.9989 \\
0.5000 & 0.5000 & $3.63 \mathrm{E}-07$ & 1.9992 & $1.88 \mathrm{E}-10$ & 2.0006 & $7.67 \mathrm{E}-07$ & 1.9994 \\
0.2500 & 0.2500 & $9.07 \mathrm{E}-08$ & 1.9994 & $4.70 \mathrm{E}-11$ & 2.0039 & $1.92 \mathrm{E}-07$ & 1.9997 \\
\hline
\end{tabular}

TABLE 3. The error and order of accuracy resulting from the FOS at time $T=1$.

\begin{tabular}{|c|c|c|c|c|c|c|c|}
\hline \multirow{2}{*}{$\begin{array}{l}t \\
\times 10^{-2}\end{array}$} & \multirow{2}{*}{$\begin{array}{l}x \\
\times 10^{-2}\end{array}$} & \multicolumn{2}{|c|}{$I(T,:)$} & \multicolumn{2}{|c|}{$B_{a}(T)$} & \multicolumn{2}{|c|}{$B_{u}(T)$} \\
\hline & & $L_{1}$ Error & Order & Error & Order & Error & Order \\
\hline 2.0000 & 2.0000 & $3.13 \mathrm{E}-02$ & & $9.32 \mathrm{E}-03$ & & 5.63E-01 & \\
\hline 1.0000 & 1.0000 & $1.55 \mathrm{E}-02$ & 1.0132 & $4.68 \mathrm{E}-03$ & 0.9953 & $2.82 \mathrm{E}-01$ & 0.9953 \\
\hline 0.5000 & 0.5000 & $7.72 \mathrm{E}-03$ & 1.0067 & $2.34 \mathrm{E}-03$ & 0.9976 & $1.41 \mathrm{E}-01$ & 0.9976 \\
\hline 0.2500 & 0.2500 & $3.85 \mathrm{E}-03$ & 1.0033 & $1.17 \mathrm{E}-03$ & 0.9988 & 7.08E-02 & 0.9988 \\
\hline$\Delta t$ & $\Delta x$ & \multicolumn{2}{|c|}{$B_{d}(T)$} & \multicolumn{2}{|c|}{$L(T)$} & \multicolumn{2}{|c|}{$S(T)$} \\
\hline$\times 10^{-2}$ & $\times 10^{-2}$ & Error & Order & Error & Order & Error & Order \\
\hline 2.0000 & 2.0000 & $9.04 \mathrm{E}-03$ & & $2.73 \mathrm{E}-01$ & & $6.05 \mathrm{E}-03$ & \\
\hline 1.0000 & 1.0000 & $4.52 \mathrm{E}-03$ & 0.9991 & $1.37 \mathrm{E}-01$ & 0.9998 & $3.03 \mathrm{E}-03$ & 0.9971 \\
\hline 0.5000 & 0.5000 & $2.26 \mathrm{E}-03$ & 0.9995 & $6.83 \mathrm{E}-02$ & 0.9999 & $1.52 \mathrm{E}-03$ & 0.9986 \\
\hline 0.2500 & 0.2500 & $1.13 \mathrm{E}-03$ & 0.9998 & 3.41E-02 & 1.0000 & 7.59E-04 & 0.9993 \\
\hline
\end{tabular}

its utility as a tool. The calibration typically involves the estimation of parameters via minimization of an objective functional. Solving such a minimization problem generally requires solving the model numerous times with high accuracy. For those endeavors, the higher order SOS is a notably better choice than the FOS.

6.2. Convergence for the full nonlinear model. We study solutions of the full model computed with both the SOS and FOS, without subpopulations (so $M=1$ ), and therefore, there is only one type of intra-host progression. The progression rate function $g$ is

$$
g\left(x, B_{a}, B_{u}, B_{d}, L ; \beta\right)=\beta(x)+\sigma_{1} \delta B_{a}+\sigma_{2} c_{1} B_{d}+\sigma_{3} \eta c_{2} L .
$$


TABLE 4. The error for the infected fish population at final time $T=1$, along with the order of accuracy and CPU time for the FOS and SOS.

\begin{tabular}{llllrlrr}
\hline$\Delta t$ & $\Delta x$ & & FOS & & \multicolumn{3}{c}{ SOS } \\
$\times 10^{-4}$ & $\times 10^{-4}$ & $L_{1}$ Error & Order & CPU(s) & \multicolumn{1}{c}{$L_{1}$ Error } & Order & CPU(s) \\
\hline 200.00 & 200.00 & $3.13 \mathrm{E}-02$ & & 0.0448 & $1.34 \mathrm{E}-04$ & & 0.0723 \\
100.00 & 100.00 & $1.55 \mathrm{E}-02$ & 1.0132 & 0.0726 & $3.13 \mathrm{E}-05$ & 2.0991 & 0.0315 \\
50.000 & 50.000 & $7.72 \mathrm{E}-03$ & 1.0067 & 0.1957 & $7.59 \mathrm{E}-06$ & 2.0463 & 0.1033 \\
25.000 & 25.000 & $3.85 \mathrm{E}-03$ & 1.0033 & 0.7461 & $1.87 \mathrm{E}-06$ & 2.0237 & 0.2342 \\
12.500 & 12.500 & $1.92 \mathrm{E}-03$ & 1.0017 & 3.0439 & $4.62 \mathrm{E}-07$ & 2.0151 & 1.1845 \\
6.2500 & 6.2500 & $9.61 \mathrm{E}-04$ & 1.0008 & 12.2517 & $1.15 \mathrm{E}-07$ & 2.0109 & 8.0171 \\
3.1250 & 3.1250 & $4.80 \mathrm{E}-04$ & 1.0004 & 48.4998 & $2.85 \mathrm{E}-08$ & 2.0068 & 78.7592 \\
1.5625 & 1.5625 & $2.40 \mathrm{E}-04$ & 1.0002 & 205.5012 & $7.10 \mathrm{E}-09$ & 2.0051 & 553.6177 \\
\hline
\end{tabular}

The functional forms of the intra-host progression rate $\beta$, the per capita shedding rate $\rho$, the diseaseinduced mortality scale factor $f_{\mu}$, the (susceptible) fish fertility rate $F$, and the human activity-induced increase in Mm populations $H$ are given in Table 5. The other parameters used in the model and their values are given in Table 6 . The parameters used in this example are not necessarily biologically meaningful, and the solutions from this parameter set are not here, or elsewhere, used to make direct inferences or predictions about any naturally occurring, or laboratory, population. However, the dynamics observed in the model solutions in this example are biologically feasible, albeit at different scales of time or population size(s).

TABLE 5. Functional forms for model parameters.

\begin{tabular}{c|c||c|c}
\hline \hline Parameter & Functional Form & Parameter & Functional Form \\
\hline$\beta(x)$ & $\tilde{\beta} x$ & $f_{\mu}(x)$ & $\exp \left(b \frac{\ln (x)-\ln \left(x_{\min }\right)}{\ln \left(x_{\max }\right)-\ln \left(x_{\min }\right)}\right)$ \\
$\rho(x)$ & $\tilde{\rho} \frac{x-x_{\min }}{x_{\max }-x_{\min }}$ & $F(Y)$ & $\frac{a Y(t)}{1+v Y(t)}$ \\
$H\left(t, B_{u}\right)$ & $\tilde{H} B_{u}$ & & \\
\hline \hline
\end{tabular}

The initial conditions are given by

$$
B_{a}(0)=0, \quad B_{u}(0)=10, \quad B_{d}(0)=0, \quad L(0)=10, \quad S(0)=10, \quad I(0, x)=0 .
$$

Notice that the initial condition of $I(0, x)=0$ is incompatible with the boundary condition

$$
I\left(0, x_{\min }\right)=\frac{1}{g\left(0, x_{\min }\right)}\left(v_{1} \delta B_{a} S+v_{2} c_{1} B_{d} S+v_{3} \eta c_{2} L S\right)
$$

at $t=0$. This generates a discontinuity (shock) in the solution along the characteristic curve emanating from the point $\left(0, x_{\min }\right)$ which can be seen in the numerical examples shown in this section.

The solutions of the model when approximated using both the SOS and FOS can be seen in Figures 1 and 2. The improved accuracy obtained from the SOS is most apparent in Figure 1, in which there are two solutions computed via the FOS for the sake of comparison. The FOS solution with the same time step $\Delta t$ and mesh discretization $\Delta x$ (the dash-dotted line) as the SOS is markedly different, most 
TABLE 6. Parameter values.

\begin{tabular}{c|c||c|c||c|c}
\hline \hline Parameter & Value & Parameter & Value & Parameter & Value \\
\hline$\mu$ & $1 / 16$ & $\gamma$ & 2 & $c_{L}$ & 50 \\
$\mu_{L}$ & $1 / 60$ & $\kappa$ & 2 & $L^{*}$ & 20 \\
$\delta$ & $1 / 500$ & $\zeta$ & 2.4 & $v_{1}$ & $1.25 \times 10^{-5}$ \\
$\tilde{\delta}$ & $1 / 500$ & $\tilde{\beta}$ & 0.4 & $\tilde{\rho}$ & 30 \\
$v_{2}$ & $1.875 \times 10^{-5}$ & $v_{3}$ & $1.25 \times 10^{-5}$ & $\eta$ & $2.4 \times 10^{3}$ \\
$\sigma_{1}$ & $2.5 \times 10^{-4}$ & $\sigma_{2}$ & $2.5 \times 10^{-4}$ & $\sigma_{3}$ & $10^{-3}$ \\
$c_{2}$ & 5 & $\alpha_{1}$ & 0 & $\alpha_{2}$ & 0 \\
$v$ & 0.05 & $a$ & 0 & $c_{1}$ & 10 \\
$b$ & 2 & $\tilde{H}$ & 0 & $\tilde{\delta}_{1}$ & $1.25 \times 10^{-6}$ \\
$\varepsilon$ & $5 \times 10^{-3}$ & & & & \\
\hline \hline
\end{tabular}

noticeably in the distribution of the infected population at a fixed time. The proportions of the infected populations at different bacterial load levels do not agree, and only when the time step and discretization of the mesh are decreased by a factor of 4 (the dashed line), do the solutions agree qualitatively. Similar conclusions about the FOS and SOS solution comparisons can be drawn from observing the time traces of the activated $\mathrm{Mm} B_{a}(t)$ and $\mathrm{Mm}$ in infected fish carcasses $B_{d}(t)$. The three solutions for the susceptible $S(t)$, unactivated $\mathrm{Mm}_{u}(t)$, and effective carrier larvae $L(t)$, all appear identical for this example.

In Figure 2 (a), the propagation of the initial cohort of infected fish (from nonzero initial carrier larvae) and the nonzero boundary condition can be seen as subsequent snapshots for the three solutions, that are also shown in Figure 1. The shock of this discontinuity can be seen as it progresses along the characteristic line gradually thereafter. The improved fidelity of the SOS solution in reproducing this behavior is also apparent here, as it was in Figure 1.

A complete view of the evolution of the infected population can be seen in Figure 2 (b), along with the corresponding infected fish of all sizes, susceptible (Figure 2 (c)) and the total fish population sizes in time (Figure 2 (d)), for the SOS solution. These, along with the time traces in Figure 1, provide a complete picture of the model dynamics for this set of parameters. The initial presence of carrier larvae $L(0)$ results in an initial increase of infected fish, that eventually progress through all possible bacterial loads $\left(\left[x_{\min }, x_{\max }\right]\right)$, and die. As they progress through infection, they shed an increasing amount of activated bacteria $B_{a}(t)$, seen in the top right panel of Figure 1. As the infected fish continue to progress and die, the bacteria in the dead fish $B_{d}(t)$ increase, as seen in the middle bottom panel of Figure 1 . Note that the rise and peak of this curve is slightly time-delayed when compared with the activated $\mathrm{Mm} B_{a}(t)$, which is reasonable according to the model's mechanisms. The declines of these two populations occur as an increasing amount of the infected fish populations progress to death. Both of these Mm populations $B_{a}(t)$ and $B_{d}(t)$ provide a source of new infections throughout the time course of the solution, albeit less than the initial cohort of infected fish. Thus the susceptible fish population decreases overall and the infected fish population first increases, and then slowly decreases, as seen in Figure 2 (c). The total population slightly decreases throughout this course of time due to the net mortality, as seen in Figure 2 (d). 

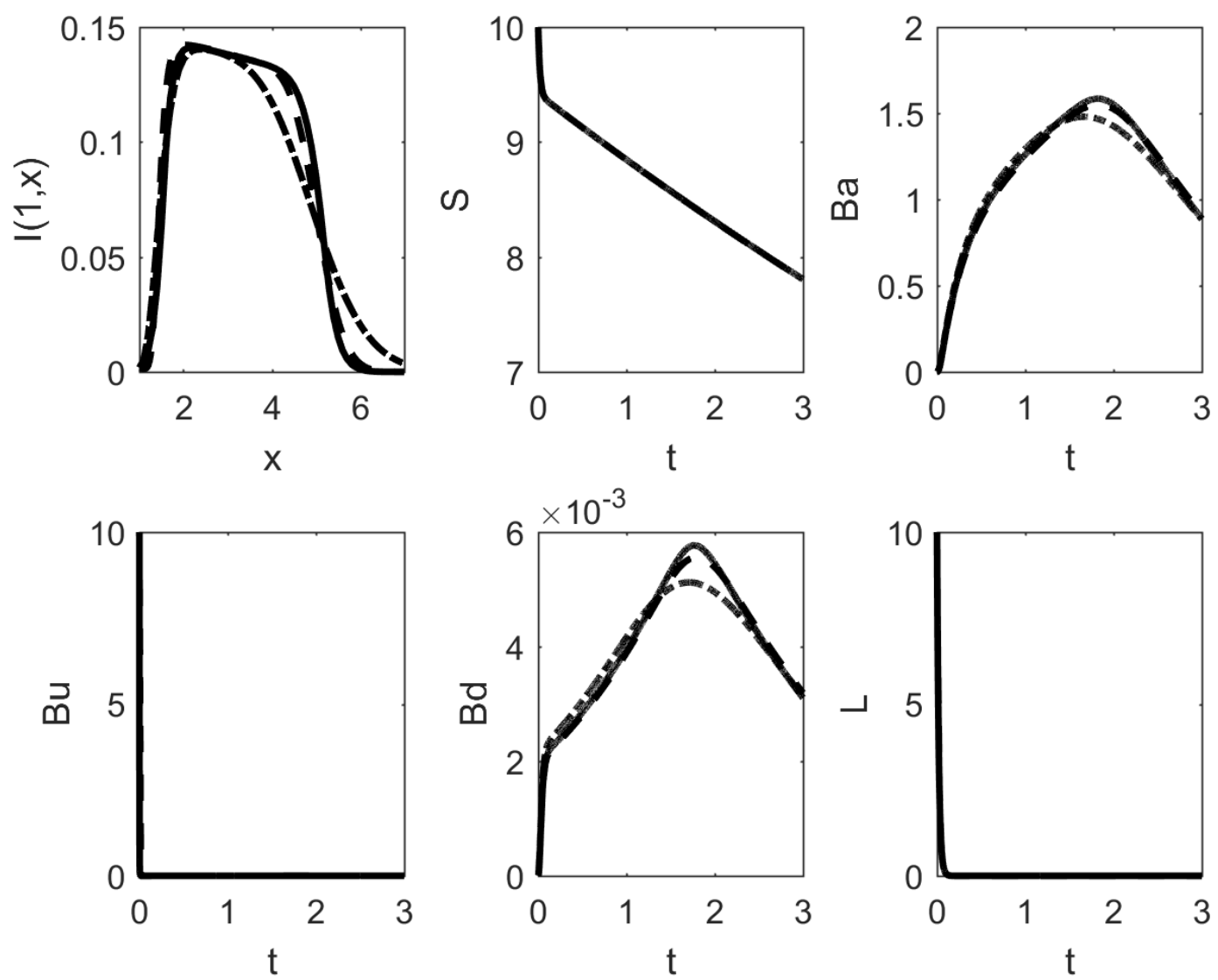

FIGURE 1. Comparison of performance of the FOS and SOS for a fully nonlinear example on $t \in[0,3]$, and their performance for the model variables in time and the infected fish density at $t=1$. The solid line represents the SOS with $\Delta t=0.0004, \Delta x=0.1$, the dash-dotted line represents the FOS with the same mesh size, and the dashed line represents the FOS solution with the discretization in both time and bacterial load reduced by a factor of $4,\left(\Delta t=\frac{0.0004}{4}\right.$ and $\left.\Delta x=\frac{0.1}{4}\right)$.

\section{CONCLUDing REMARKS}

We have established a second order high resolution finite difference scheme for a bacterial loadstructured model of Mycobacterium marinum in aquatic animals. The finite difference approximation is easily implemented, which makes this approach more attractive for this model than others (e.g., finite element methods, integration along characteristics, etc.). We established convergence of the difference approximations to a unique weak solution with bounded total variation. We numerically demonstrated the expected order of convergence for a simplified version of the model to its known exact solution. For this scenario, we compared the order of convergence and the computational efficiency with a first order scheme (given in [4]) that was originally used to compute solutions for a different version of this model. Solutions of a full model were compared using both the first order and second order scheme, and it is evident that the higher order method results in a more accurate approximation in substantially less 
(a)

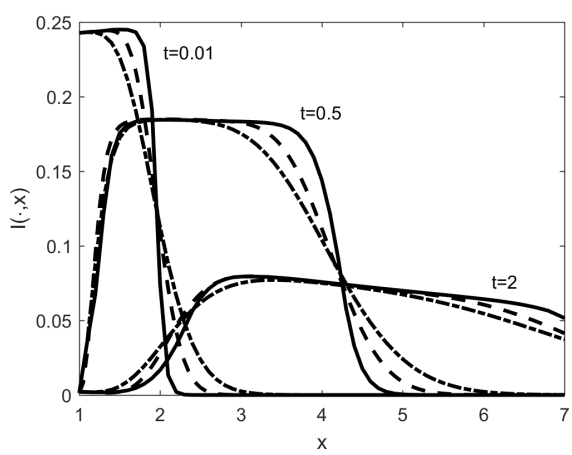

(c)

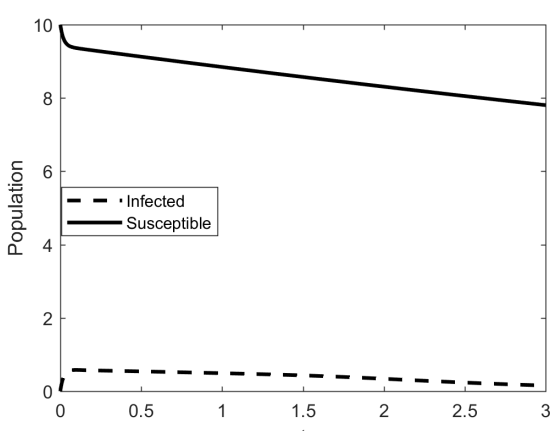

(b)

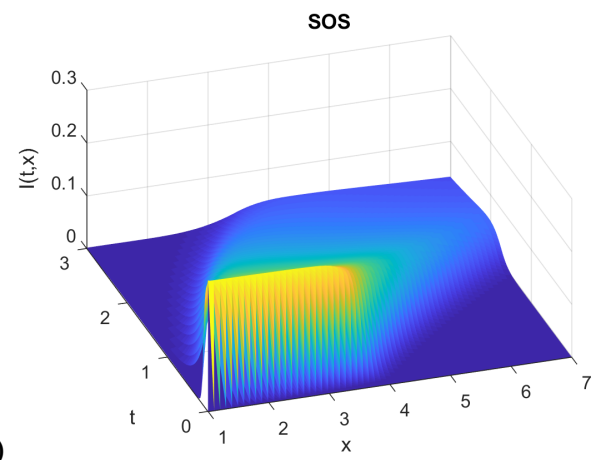

(d)

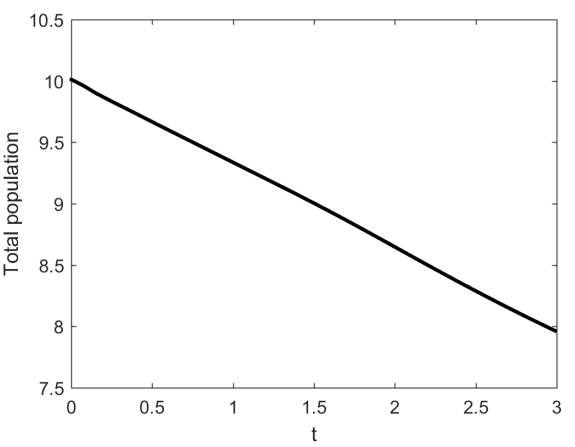

FIGURE 2. (a) The infected population at time step $t=0.01,0.5$ and 2 using both the FOS and SOS methods. The solid line represents the SOS with $\Delta t=0.0004, \Delta x=0.1$, the dash-dotted line represents the FOS with the same mesh size, and the dashed line represents the FOS with mesh size reduced 4 times on both $\Delta x$ and $\Delta t$. (b) The density of infected individuals for $t \in[0,3]$ and $x \in[1,7]$ using the SOS. (c) The total infected and susceptible population for $t \in[0,3]$ using the SOS. (d) The total population of the host for $t \in[0,3]$ using the SOS.

computational time. Accurate approximations are of particular importance in practice, as there may very well be important implications for any predictions or inferences regarding underlying mechanisms from approximated model solutions. Furthermore, when dealing with parameter estimation problems (e.g., $[1,6])$, one may need to solve the model numerous times until an optimal estimated parameter value is obtained. In such applications time efficiency and accuracy of the numerical scheme used is critical.

\section{REFERENCES}

[1] A.S. Ackleh, S. Aizicovici, S. Reich, Parameter identification in nonlocal nonlinear evolution equations, Numer. Funct. Anal. Optim. 21 (2000), 553-570.

[2] A.S. Ackleh, M.L. Delcambre, K.L. Sutton, D. Ennis, Structured models for the spread of Mycobacterium marinum: foundations for a numerical approximation scheme, Math. Biosci. Eng. 11 (2014), 679-721.

[3] A. S. Ackleh, M. L. Delcambre, K. L. Sutton, A second-order high-resolution finite difference scheme for a size-structured model for the spread of Mycobacterium marinum, J. Biol. Dyn. 9 (2014), 156-187.

[4] A. S. Ackleh, K. L. Sutton, K. N. Mutoji, A. Mallick, D. G. Ennis, A Structured Model for the Transmission Dynamics of Mycobacterium marinum between Aquatic Animals, J. Biol. Syst. 22 (2014), 29-60.

[5] A. S. Ackleh, K. L. Sutton, Disparate Disease Outcomes in Chronic Infection: the Role of Intra-Host Variability, Int. J. Pure Appl. Math.116 (2017), 343-352. 
[6] H.T. Banks, K. Kunisch, Estimation Techniques for Distributed Parameter Systems, Birkhauser, Boston, 1989.

[7] G. W. Broussard, D. G. Ennis, Mycobacterium marinum produces long-term chronic infections in medaka: a new animal model for studying human tuberculosis, Comp. Biochem. Physiol. Toxicol. Pharmacol. CBP, 145 (2007), 45-54.

[8] C. L. Cosma, D. R. Sherman, L. Ramakrishnan, The secret lives of the pathogenic mycobacteria, Ann. Rev. Microbiology, 57 (2003), 641-676.

[9] J. M. Davis, H. Clay, J. L. Lewis, N. Ghori, P. Herbomel, L. Ramakrishnan, Real-time visualization of MycobacteriumMacrophage interactions leading of initiation of granuloma formation in zebrafish embryos, Immunity, 17 (2002), 693702.

[10] A. Diamant, A. Banet, M. Ucko, A. Colorni, W. Knibb, H. Kvitt, Mycobacteriosis in wild rabbitfish Siganus rivulatus associated with cage farming in the Gulf of Eilat, Red Sea, Dis. Aquat. Org. 39 (2000), 211-219.

[11] S. H. El-Etr, L. Yan, J. D. Cirillo, Fish monocytes as a model for mycobacterial host-pathogen interactions, Infect. Immun. 69 (2001), 7310-7317.

[12] R. E. Gozlan, S. St-Hilaire, S. W. Feist, P. Martin, M. L. Kent, Biodiversity: Disease threat to European fish, Nature, 435 (2005), 1046.

[13] R. P. Hedrick, T. McDowell, J. Groff, Mycobacteriosis in cultured striped bass from California, J Wild Dis. 23 (1987), 391-395.

[14] J. M. Jacobs, C. B. Stine, A. M. Baya, M. L. Kent, A review of mycobacteriosis in marine fish, J. Fish Dis. 32 (2009), 119-130.

[15] A. S. Kane, C. B. Stine, L. Hungerford, M. Matsche, C. Driscoll, A. M. Baya, Mycobacteria as environmental portent in Chesapeake Bay fish species, Emerg. Infect. Dis. 13 (2007), 329-331.

[16] Miltner E, Daroogheh K, Mehta PK, Cirillo SL, Cirillo JD, Bermudez LE, Identification of Mycobacterium avium genes that affect invasion of the intestinal epithelium, Infect. Immun. 73 (2005), 4214-4221.

[17] B. Petrini, Mycobacterium marinum: ubiquitous agent of waterborne granulomatous skin infections, Eur. J. Clin. Microbiol. Infect. Dis. 25 (2006), 609-613.

[18] M. G. Prouty, N. E. Correa, L. P. Barker, P. Jagadeeswaran, K. E. Klose, Zebrafish-Mycobacterium marinum model for mycobacterial pathogenesis, FEMS Microbiol. Lett. 225 (2003), 177-182.

[19] M. C. Raviglione, D. E. Snider Jr, A. Kochi, Global epidemiology of tuberculosis: morbidity and mortality of a worldwide epidemic, J. Am. Med. Assoc. 273 (1995), 220-226.

[20] M. Reed, B. Simon, Methods of Modern Mathematical Physis I: Functional Analysis, Academic Press, New York, 1980.

[21] J. Smoller, Shock Waves and Reaction-Diffusion Equation, Springer-Verlag, New York, 2007.

[22] C. B. Stine, A. S. Kane, A. M. Baya, Mycobacteria isolated from Chesapeake Bay fishes, J. Fish Dis. 33 (2009), 39-46.

[23] A. M. Talaat, R. Reimschuessel, S. S. Wasserman, M. Trucksis, Goldfish, Carassius auratus, a novel animal model for the study of Mycobacterium marinum pathogenesis, Infect. Immun. 66 (1998), 2938-2942.

[24] World Health Organization, Global tuberculosis report 2016, WHO press, Geneva, Switzerland, 2016.

[25] B. A. Wilson, A. A. Salyers, D. D. Whitt, M. E. Winkler, Bacterial Pathogenesis: a Molecular Approach, 3rd Edition, ASM Press, Washington, DC, 2010. 